\title{
FORMATION OF SINGULARITIES FOR VISCOSITY SOLUTIONS OF HAMILTON-JACOBI EQUATIONS
}

\author{
SHYUICHI IZUMIYA \\ Department of Mathematics, Faculty of Science, Hokkaido University \\ Sapporo 060, Japan \\ GEORGIOS T. KOSSIORIS \\ Department of Mathematics, University of Crete \\ 71409 Iraklion, Crete, Greece
}

1. Introduction. In this note we study the generation and propagation of singularities (shock waves) of the solution of the Cauchy problem for Hamilton-Jacobi equations

$$
\left\{\begin{array}{l}
\frac{\partial y}{\partial t}+H\left(t, x_{1}, \ldots, x_{n}, \frac{\partial y}{\partial x_{1}}, \ldots, \frac{\partial y}{\partial x_{n}}\right)=0 \\
y\left(0, x_{1}, \ldots, x_{n}\right)=\phi\left(x_{1}, \ldots, x_{n}\right)
\end{array}\right.
$$

where $H$ and $\phi$ are $C^{\infty}$-functions.

Hamilton-Jacobi equations play an important role in various fields e.g., calculus of variations (see e.g., [21]), optimal control theory (see e.g., [9]) and differential games (see e.g., [8] and references cited therein).

For small time $t$ the solution of $(\mathrm{P})$ is classically determined using the characteristic method. The geometric solution $y$ of $(\mathrm{P})$ has been defined in ([13], [14]) in the framework of one-parameter Legendrian unfoldings and it is constructed by the method of characteristics. Although $y$ is initially smooth there is in general a critical time beyond which characteristics cross. The geometric solution past the critical time is multi-valued, that is, singularities appear. The classification of singularities of $y$ has been studied in [13] (see also [15]) In Section 2 we give a survey on the geometric framework ([13], [15], [16]).

The theory of viscosity solutions (see [5]) has provided the right weak setting for the study of $(\mathrm{P})$. Existence and uniqueness of the solution of $(\mathrm{P})$ in the viscosity sense have been established in [6]. The single-valued viscosity solution is continuous and coincides with the smooth geometric solution until the first critical time. After the characteristics cross, the viscosity solution develops shock waves, i.e., curves across which the gradient

1991 Mathematics Subject Classification: 35L67, 58C27.

The paper is in final form and no version of it will be published elsewhere. 
of the viscosity solution is discontinuous. The shock surfaces are referred to as singular surfaces in the literature of optimal control and differential games (see e.g., [3], [12]).

The method of constructing the weak solution by selecting the proper single-valued branch was introduced by Tsuji ([22], [23]) for Hamilton-Jacobi equations. Nakane in [20] has constructed the weak semi-concave solution past the first critical time in case that $H$ is convex with respect to $\nabla y=\left(\frac{\partial y}{\partial x_{1}}, \ldots, \frac{\partial y}{\partial x_{n}}\right)$. The case of scalar conservation laws in $\mathbb{R}^{n}$ past the first critical time has been studied by Nakane in [19]. In [4] Bogaevskiu has shown that the potential solution of the Burgers system with vanishing viscosity is given by the minimum function of a certain family of smooth functions and given a classification for $n=1,2,3$. It corresponds to the viscosity solution of the Hamilton-Jacobi equation when the Hamiltonian is given by $H\left(p_{1}, \ldots, p_{n}\right)=\frac{1}{2} p_{1}^{2}+\ldots+\frac{1}{2} p_{n}^{2}$.

The viscosity solution of $(\mathrm{P})$ for general Hamiltonian in a neighborhood of the first critical time has been constructed in [18] (see also [17], [20]) by selecting a continuous single-valued branch of the graph of the geometric solution in which the shock curves of the weak solution correspond to the intersection of the branches of the graph of the multi-valued geometric solution. In order to study the evolution of the shock curves we follow the evolution of the intersections of the branches defining the shock. After that we solve local Riemann problems for each stage.

Here, we give proofs for some of the results. Further discussions will appear elsewhere. All maps considered here are of class $C^{\infty}$ unless stated otherwise.

2. Geometric solutions. In this section we give a survey on the geometric framework and present the necessary notations which was described in ([13], [15], [16]).

Let $J^{1}\left(\mathbb{R}^{n}, \mathbb{R}\right)$ be the 1 -jet bundle of functions of $n$-variables which may be considered as $\mathbb{R}^{2 n+1}$ with a natural coordinate system $\left(x_{1}, \ldots, x_{n}, y, p_{1}, \ldots, p_{n}\right)$, where $\left(x_{1}, \ldots, x_{n}\right)$ is a coordinate system of $\mathbb{R}^{n}$. We also have a natural projection $\pi: J^{1}\left(\mathbb{R}^{n}, \mathbb{R}\right) \rightarrow \mathbb{R}^{n} \times \mathbb{R}$ given by $\pi(x, y, p)=(x, y)$.

An immersion germ $i:\left(L_{0}, u_{0}\right) \rightarrow J^{1}\left(\mathbb{R}^{n}, \mathbb{R}\right)$ is said to be a Legendrian immersion germ (i.e., Legendrian submanifold germ) if $\operatorname{dim} L=n$ and $i^{*} \theta=0$, where $\theta=$ $d y-\sum_{i=1}^{n} p_{i} \cdot d x_{i}$. The image of $\pi \circ i$ is called the wave front set of $i$ and it is denoted by $W(i)$. We also consider the 1 -jet bundle $J^{1}\left(\mathbb{R} \times \mathbb{R}^{n}, \mathbb{R}\right)$ and the canonical 1form $\Theta$ on that space. Let $\left(t, x_{1}, \ldots, x_{n}\right)$ be a canonical coordinate system on $\mathbb{R} \times \mathbb{R}^{n}$ and $\left(t, x_{1}, \ldots, x_{n}, y, s, p_{1}, \ldots, p_{n}\right)$ the corresponding coordinate system on $J^{1}\left(\mathbb{R} \times \mathbb{R}^{n}, \mathbb{R}\right)$. Then, the canonical 1 -form is given by $\Theta=d y-\sum_{i=1}^{n} p_{i} \cdot d x_{i}-s \cdot d t=\theta-s \cdot d t$.

We define the natural projection $\Pi: J^{1}\left(\mathbb{R} \times \mathbb{R}^{n}, \mathbb{R}\right) \rightarrow\left(\mathbb{R} \times \mathbb{R}^{n}\right) \times \mathbb{R}$ by $\Pi(t, x, y, s, p)=$ $(t, x, y)$. We call the above 1 -jet bundle an unfolded 1-jet bundle.

A Hamilton-Jacobi equation is defined to be a hypersurface

$(\mathrm{G}-\mathrm{H}-\mathrm{J}) \quad E(H)=\left\{(t, x, y, s, p) \in J^{1}\left(\mathbb{R} \times \mathbb{R}^{n}, \mathbb{R}\right) \mid s+H(t, x, p)=0\right\}$

in $J^{1}\left(\mathbb{R} \times \mathbb{R}^{n}, \mathbb{R}\right)$. A geometric (multi-valued) solution of $E(H)$ is a Legendrian submanifold $L$ in $J^{1}\left(\mathbb{R} \times \mathbb{R}^{n}, \mathbb{R}\right)$ lying in $E(H)$. In this case the wave front set $W(i)$ is "the graph" of the geometric solution which is generally a hypersurface with singularities. 
In order to study $(\mathrm{P})$ we need the following framework: For any $c \in(\mathbb{R}, 0)$, we define

$$
E(H)_{c}=\left\{(c, x, y,-H(c, x, p), p) \mid(x, y, p) \in J^{1}\left(\mathbb{R}^{n}, \mathbb{R}\right)\right\} .
$$

Then, $E(H)_{c}$ is a $(2 n+1)$-dimensional submanifold of $J^{1}\left(\mathbb{R} \times \mathbb{R}^{n}, \mathbb{R}\right)$ and $\Theta_{c}=$ $\Theta \mid E(H)_{c}=d z-\sum_{i=1}^{n} p_{i} d x_{i}$ gives a contact structure on $E(H)_{c}$. We define a mapping $\iota_{c}: J^{1}\left(\mathbb{R}^{n}, \mathbb{R}\right) \rightarrow E(H)_{c}$ by $\iota_{c}(x, y, p)=(c, x, y,-H(c, x, p), p)$. The mapping $i_{c}$ is a contact diffeomorphism and the following diagram is commutative:

$$
\begin{array}{ccc}
J^{1}\left(\mathbb{R}^{n}, \mathbb{R}\right) & \stackrel{\iota_{c}}{\longrightarrow} & E(H)_{c} \\
\pi \downarrow & & \downarrow \pi_{c} \\
\mathbb{R}^{n} \times \mathbb{R} & = & \mathbb{R}^{n} \times \mathbb{R}
\end{array}
$$

We say that a geometric Cauchy problem (with initial condition $L^{\prime}$ ) associated with the time parameter $(G C P T)$ is given for an equation $E(H)$ if there is given an $n$-dimensional submanifold $i: L^{\prime} \subset E(H)$ with $i^{*} \Theta=0$ and $i\left(L^{\prime}\right) \subset E(H)_{c}$ for some $c \in(\mathbb{R}, 0)$. Since $X_{H} \notin T E(H)_{c}$, we have $X_{H} \notin T L^{\prime}$, where $X_{H}$ is the characteristic vector field given by

$$
X_{H}=\frac{\partial}{\partial t}+\sum_{i=1}^{n} \frac{\partial H}{\partial p_{i}} \frac{\partial}{\partial x_{i}}+\left(\sum_{i=1}^{n} p_{i} \frac{\partial H}{\partial p_{i}}-H\right) \frac{\partial}{\partial y}-\frac{\partial H}{\partial t} \frac{\partial}{\partial s}-\sum_{i=1}^{n} \frac{\partial H}{\partial x_{i}} \frac{\partial}{\partial p_{i}} .
$$

By using the classical characteristic method, we can show that there exists a unique geometric solutions around $L^{\prime}$.

We remark that Cauchy problem (P) is a GCPT. The initial submanifold is given by

$$
L_{\phi, 0}=\left\{\left(0, x, \phi(x),-H\left(0, x, \frac{\partial \phi}{\partial x}\right), \frac{\partial \phi}{\partial x}\right) \mid x \in \mathbb{R}^{n}\right\} \subset E(H)_{0} .
$$

The problem of studying the singularities of the graph of the geometric solution is formulated as follows:

Geometric Problem. Classify the generic bifurcations of wave fronts of

$$
\pi_{t} \mid: L \cap E(H)_{t} \rightarrow \mathbb{R}^{n} \times \mathbb{R}
$$

with respect to the parameter $t$ (i.e., the generic bifurcations of wave fronts of geometric solutions along the time parameter).

Following [16], in order to study the singularities of the geometric solution we identify geometric solutions with one-parameter Legendrian unfoldings. Let $R$ be an $(n+1)$ dimensional smooth manifold, $\mu:\left(R, u_{0}\right) \rightarrow\left(\mathbb{R}, t_{0}\right)$ be a submersion germ and $\ell$ : $\left(R, u_{0}\right) \rightarrow J^{1}\left(\mathbb{R}^{n}, \mathbb{R}\right)$ be a smooth map germ. We say that the pair $(\mu, \ell)$ is a Legendrian family if $\ell_{t}=\ell \mid \mu^{-1}(t)$ is a Legendrian immersion germ for any $t \in\left(\mathbb{R}, t_{0}\right)$. Then we have the following simple but very important lemma.

LEMMA 2.1 Let $(\mu, \ell)$ be a Legendrian family. Then there exist a unique element $h \in C_{u_{0}}^{\infty}(R)$ such that $\ell^{*} \theta=h \cdot d \mu$, where $C_{u_{0}}^{\infty}(R)$ is the ring of smooth function germs at $u_{0}$.

Define a map germ $\mathcal{L}:\left(R, u_{0}\right) \rightarrow J^{1}\left(\mathbb{R} \times \mathbb{R}^{n}, \mathbb{R}\right)$ by

$$
\mathcal{L}(u)=(\mu(u), x \circ \ell(u), y \circ \ell(u), h(u), p \circ \ell(u)) .
$$


We can easily show that $\mathcal{L}$ is a Legendrian immersion germ. If we fix 1 -forms $\Theta$ and $\theta$, the Legendrian immersion germ $\mathcal{L}$ is uniquely determined by the Legendrian family $(\mu, \ell)$. We call $\mathcal{L}$ a Legendrian unfolding associated with the Legendrian family $(\mu, \ell)$.

In order to study the evolution of the shock waves of the viscosity solutions of $(\mathrm{P})$, we have to classify the generic types of the appearing singularities i.e., how a singularity is generated, how one type can change into another and how different types of singularities interact. We study how various branches of the multi-valued graph $W_{t}=$ $\left(\{t\} \times \mathbb{R}^{n} \times \mathbb{R}\right) \cap W(i)$ intersecting at a point bifurcate in time for an arbitrary Hamiltonian $H(t, x, p)$ in [15]. We classify the bifurcations of the branches of the graph by classifying the bifurcations of singularities of multi-Legendrian unfoldings which are expressed in terms of multi-germs.

Let $\mathcal{L}_{i}:\left(R, u_{0}\right) \rightarrow\left(J^{1}\left(\mathbb{R} \times \mathbb{R}^{n}, \mathbb{R}\right), z_{i}\right)(i=1, \ldots, r)$ be Legendrian unfoldings with $\Pi\left(z_{i}\right)=0$ where $z_{1}, \ldots, z_{r}$ are distinct. We call $\left(\mathcal{L}_{1}, \ldots, \mathcal{L}_{r}\right)$ a multi-Legendrian unfolding. Let $\left(\mathcal{L}_{1}, \ldots, \mathcal{L}_{r}\right)$ and $\left(\mathcal{L}_{1}^{\prime}, \ldots, \mathcal{L}_{r}^{\prime}\right)$ be multi-Legendrian unfoldings. We say that these are $P_{(r)}$-Legendrian equivalent if there exist contact diffeomorphism germs

$$
K_{i}:\left(J^{1}\left(\mathbb{R} \times \mathbb{R}^{n}, \mathbb{R}\right), z_{i}\right) \rightarrow\left(J^{1}\left(\mathbb{R} \times \mathbb{R}^{n}, \mathbb{R}\right), z_{i}^{\prime}\right) \quad(i=1, \ldots, r)
$$

of the form $K_{i}(t, x, y, s, p)=\left(\phi_{1}(t), \phi_{2}(t, x, y), \phi_{3}(t, x, y), \phi_{4}^{i}(t, x, y, s, p), \phi_{5}^{i}(t, x, y, s, p)\right)$ and a diffeomorphism germ $\Psi:\left(R, u_{0}\right) \rightarrow\left(R, u_{0}^{\prime}\right)$ such that $K_{i} \circ \mathcal{L}_{i}=\mathcal{L}_{i}^{\prime} \circ \Psi$ for any $i=$ $1, \ldots, r$. It is clear that if two multi-Legendrian unfoldings are $P_{(r)}$-Legendrian equivalent, then there exists a diffeomorphism germ $\Phi:\left(\mathbb{R} \times\left(\mathbb{R}^{n} \times \mathbb{R}\right), 0\right) \rightarrow\left(\mathbb{R} \times\left(\mathbb{R}^{n} \times \mathbb{R}\right), 0\right)$ of the form $\Phi(t, x, y)=\left(\phi_{1}(t), \phi_{2}(t, x, y), \phi_{3}(t, x, y)\right)$ such that $\Phi\left(\cup_{i=1}^{r} W\left(\mathcal{L}_{i}\right)\right)=\cup_{i=1}^{r} W\left(\mathcal{L}_{i}\right)$. Thus the above equivalence describes how bifurcations of wavefronts (i.e. graphs of solutions) interact. We can define the notion of stability with respect to the $P_{(r)}$-Legendrian equivalence in the same way as for the ordinary Legendrian stability (see [1], [24]). Motivated by Arnol'd-Zakalyukin's theory ([1], [24]), we can construct multi-generating families of multi-Legendrian unfoldings and give a classification of $P_{(r)}$-Legendrian stable Legendrian unfoldings by using the classification of multi-families of function germs in Zakalyukin [24]. We get a list of classifications for $n=1,2,3$ in [15]. However, we only present the list of classifications for $n=1$. For the case $n=2,3$, see [15].

TheOrem 2.2 [15]. Suppose that $n=1$. Then a generic multi-Legendrian unfolding is $P_{(r)}$-Legendrian equivalent to one of the multi-Legendrian unfoldings in the following list:

$$
\begin{array}{ll} 
& { }^{0} A_{1}:(t, u, 0,0,0) ; \\
-r=1: & { }^{0} A_{2}:\left(t, 3 u^{2}, 2 u^{3}, 0, u\right) ; \\
& { }^{1} A_{3}:\left(t, 4 u^{3}+2 u t, 3 u^{4}+u^{2} t,-u^{2}, u\right) . \\
& { }^{0}\left({ }^{0} A_{1}{ }^{0} A_{1}\right):((t, u,-u, 0,-1),(t, u, u, 0,1)) ; \\
-r=2: & { }^{1}\left({ }^{0} A_{1}{ }^{0} A_{1}\right):\left(\left(t, u, t \pm u^{2}, 1, \pm 2 u\right),(t, u, 0,0,0)\right) ; \\
& { }^{1} A_{2}{ }^{0} A_{1}:\left(\left(t, 3 u^{2}-t, 2 u^{3}, u, u\right),(t, u,-u, 0,-1)\right) . \\
\text { - } r=3: & { }^{0} A_{1}{ }^{0} A_{1}{ }^{0} A_{1}:((t, u, t-u, 1,-1),(t, u, 0,0,0),(t, u, u, 0,1)) .
\end{array}
$$

When we consider the geometric solution, we can get rid of the germ ${ }^{1}\left({ }^{0} A_{1}{ }^{0} A_{1}\right)$ from the above list because the geometric solution is a one-to-one immersions into the unfolded 1 -jet space. 
On the other hand, we have to identify geometric solutions with one-parameter Legendrian unfoldings in locally, so that we prove the following realization theorems.

TheOREM 2.3 [13]. (1) The local solution of the GCPT for the Hamilton-Jacobi equation $(G-H-J)$ is a Legendrian unfolding $\mathcal{L}:\left(\mathbb{R} \times \mathbb{R}^{n}, 0\right) \rightarrow J^{1}\left(\mathbb{R} \times \mathbb{R}^{n}, \mathbb{R}\right)$.

(2) Let $\mathcal{L}:\left(\mathbb{R} \times \mathbb{R}^{n}, 0\right) \rightarrow J^{1}\left(\mathbb{R} \times \mathbb{R}^{n}, \mathbb{R}\right)$ be a Legendrian unfolding associated with $\left(\pi_{1}, \ell\right)$. Then there exists a $C^{\infty}$-function germ $H\left(t, x_{1}, \ldots, x_{n}, p_{1}, \ldots, p_{n}\right)$ such that $\mathcal{L}$ is a local solution of the generalized Cauchy problem associated with the time parameter for the Hamilton-Jacobi equation $(G-H-J)$, where the initial condition is given by $\ell(0, u)$.

The above theorem guarantees that the class of Legendrian unfoldings supplies the correct class to describe the geometric solutions of (GCPT) for Hamilton-Jacobi equations. Thus, generic results for the singularities of Legendrian unfoldings can be translated to generic results in the class of all Hamiltonians and all initial conditions. However, we also have to concern ourselves with what are the types of singularities that the geometric solution to a given Hamilton-Jacobi equation might exhibit. For this purpose, we need a kind of non-degeneracy condition on the Hamiltonian function. We say that a Hamiltonian function $H(t, x, p)$ is non-degenerate at $\left(t_{0}, x_{0}, p_{0}\right)$ if $\frac{\partial^{2} H}{\partial p_{i} \partial p_{j}}\left(t_{0}, x_{0}, p_{0}\right) \neq 0$ for some $1 \leq i, j \leq n$. This condition is weaker than the condition that $H(t, x, p)$ is convex (or concave) with respect to $\left(p_{1}, \ldots, p_{n}\right)$-variables at $\left(t_{0}, x_{0}, p_{0}\right)$ for $n \geq 2$. The following theorem is a realization theorem for generic singularities for a given Hamilton-Jacobi equation.

TheOREM $2.4([15],[16])$. Let $H(t, x, p)$ be a non-degenerate Hamiltonian function germ at $\left(t_{0}, x_{0}, p_{0}\right)$ and $\mathcal{L}:\left(R, u_{0}\right) \rightarrow\left(J^{1}\left(\mathbb{R} \times \mathbb{R}^{n}, \mathbb{R}\right),\left(t_{0}, x_{0}, y_{0}, s_{0}, p_{0}\right)\right)$ be a $P_{(1)}$ Legendrian stable Legendrian unfolding associated with $(\mu, \ell)$. Then there exists a Legendrian unfolding $\mathcal{L}^{\prime}$ which is a geometric solution of the Hamilton-Jacobi equation $s+H(t, x, p)=0$ such that $\mathcal{L}$ and $\mathcal{L}^{\prime}$ are $P_{(1)}$-Legendrian equivalent.

We remark that ${ }^{1} A_{3}$ singularity (even for general $n$ ) describes how the singularity appears from a smooth solution. These are $P_{(1)}$-Legendrian stable Legendrian unfoldings, so that these can be realized as geometric solutions at the non-degenerate point for a given Hamilton-Jacobi equation. We can also specify the point at where the ${ }^{1} A_{3}$-singularity appears.

THEOREM $2.5[16]$. If an ${ }^{1} A_{3}$-singularity appears at $\left(t_{0}, x_{0}, p_{0}\right)$, then $H(t, x, p)$ is nondegenerate at $\left(t_{0}, x_{0}, p_{0}\right)$.

3. Viscosity solutions. The viscosity solutions for nonlinear equations of first order have been introduced by Crandall and Lions [6]. Such solutions need not be differentiable everywhere, as the only regularity required in the definition is that of continuity. The function $y_{\mathfrak{v}} \in C(\mathcal{O})$ is a viscosity solution of

$$
(\mathrm{H}-\mathrm{J}) \quad \frac{\partial y}{\partial t}+H\left(t, x, \frac{\partial y}{\partial x_{1}}, \ldots, \frac{\partial y}{\partial x_{n}}\right)=0
$$


in the open domain $\mathcal{O} \subset \mathbb{R}^{+} \times \mathbb{R}^{n}$ provided

$$
\frac{\partial \psi}{\partial t}(t, x)+H\left(t, x, \frac{\partial \psi}{\partial x_{1}}(t, x), \ldots, \frac{\partial \psi}{\partial x_{n}}(t, x)\right) \leq 0, \quad(\text { resp. } \geq 0)
$$

for any $\psi \in C^{1}(\mathcal{O})$ for which $y_{\mathfrak{v}}-\psi$ attains a local maximum (resp. local minimum) at the point $(t, x) \in \mathcal{O}$. The function $y_{\mathfrak{v}} \in C\left([0, \infty) \times \mathbb{R}^{n}\right)$ is a viscosity solution of the Cauchy problem $(P)$ if and only if it is a viscosity solution of $(\mathrm{H}-\mathrm{J})$ in the domain $(0, \infty) \times \mathbb{R}^{n}$ and satisfies the initial condition

$$
\lim _{t \rightarrow 0+} y_{\mathfrak{v}}(t, x)=\phi(x) .
$$

The above inequality will be referred to as the viscosity criterion at the point $(t, x)$. We next state the viscosity criterion in a form which is more useful for the construction of the solution. To this end, assume that $\mathcal{O} \subset(0, \infty) \times \mathbb{R}^{n}$ is open and that there is a smooth hypersurface $\Gamma$ of $\mathbb{R}^{+} \times \mathbb{R}^{n}$, which divides $\mathcal{O}$ into two open sets $\mathcal{O}^{+}$and $\mathcal{O}^{-}$, $\mathcal{O}=\Gamma \cup \mathcal{O}^{+} \cup \mathcal{O}^{-}$. Then we have the following theorem.

THEOREM 3.1. Let $y_{\mathfrak{v}} \in C(\mathcal{O})$ and $y_{\mathfrak{v}}=y_{\mathfrak{v}}^{+}$in $\mathcal{O}^{+} \cup \Gamma, y_{\mathfrak{v}}=y_{\mathfrak{v}}^{-}$in $\mathcal{O}^{-} \cup \Gamma$ where $y_{\mathfrak{v}}^{ \pm} \in C^{1}\left(\mathcal{O}^{ \pm} \cup \Gamma\right)$. Then $y_{\mathfrak{v}}$ is a viscosity solution of $(\mathrm{H}-\mathrm{J})$ in $\mathcal{O}$ if and only if the following conditions hold:

(a) $y_{\mathfrak{v}}^{+}$and $y_{\mathfrak{v}}^{-}$are classical solutions of $(H-J)$ in $\mathcal{O}^{+}$and $\mathcal{O}^{-}$respectively,

(b) If the vector $\tilde{\eta}=\left(H\left(t, x, \frac{\partial y^{+}}{\partial x}\right)-H\left(t, x, \frac{\partial y^{+}}{\partial x}\right),-\left(\frac{\partial y^{+}}{\partial x_{1}}-\frac{\partial y^{-}}{\partial x_{1}}, \ldots, \frac{\partial y^{+}}{\partial x_{n}}-\frac{\partial y^{-}}{\partial x_{n}}\right)\right)$ points into $\mathcal{O}^{+}$, then

$$
\begin{aligned}
H\left(t, x,(1-\lambda) \frac{\partial y_{\mathfrak{v}}^{+}}{\partial x}\right. & \left.+\lambda \frac{\partial y_{\mathfrak{v}}^{-}}{\partial x}\right) \\
& -(1-\lambda) H\left(t, x, \frac{\partial y_{\mathfrak{v}}^{+}}{\partial x}\right)-\lambda H\left(t, x, \frac{\partial y_{\mathfrak{v}}^{-}}{\partial x}\right) \leq 0 \quad(\text { resp. } \geq 0),
\end{aligned}
$$

where $\lambda \in[0,1]$. In particular, the graph of $H$ lies respectively below or above the line segment joining the points $\left(H\left(t, x, \frac{\partial y_{\mathrm{v}}^{+}}{\partial x}\right), \frac{\partial y_{\mathrm{v}}^{+}}{\partial x}\right)$ and $\left(H\left(t, x, \frac{\partial y_{\mathrm{v}}^{-}}{\partial x}\right), \frac{\partial y_{\mathrm{v}}^{-}}{\partial x}\right)$.

The proof of Theorem 3.1 is given in ([17], [18]) as a direct application of Theorem 1.3 in [7]. The condition (b) will be referred to in the sequel as the viscosity criterion. The hypersurface $\Gamma$ in the neighbourhood of which $y_{\mathfrak{v}}$ has the properties specified in the above theorem is the shock surface. If the Hamiltonian is uniformly convex (or concave), we can automatically construct viscosity solutions from our normal forms, so that we can easily draw the pictures of shock surfaces for lower dimensional cases. In [4] Bogaevskiu has shown that the potential solution of the Burgers system with vanishing viscosity is given by the minimum function of a certain family of smooth functions. It corresponds to the viscosity solution of the Hamilton-Jacobi equation when the Hamiltonian is given by $H\left(p_{1}, \ldots p_{n}\right)=\frac{1}{2} p_{1}^{2}+\ldots+\frac{1}{2} p_{n}^{2}$. He has drawn the pictures of shocks for this case. Our pictures are same as his pictures, so we do not present these in here (see [4]).

On the other hand, Bogaevskiu used the Florin-Hopf-Cole method ([10], [11]) to detect the solution and it works only for the Burgers system. Here, we prove the analogous statement as the Bogaevski1's assertion in the case when the Hamiltonian $H\left(p_{1}, \ldots, p_{n}\right)$ is convex and depends only on the momentum. In this case we apply Bardi-Evans' result[2] 
to our situations in stead of Florin-Hopf-Cole method. The geometric solution for $(\mathrm{P})$ is given by

$$
L_{\phi, t}=\left\{(t, x(t, u), y(t, u),-H(p(t, u)), p(t, u)) \mid u \in \mathbb{R}^{n}\right\}
$$

where

$$
\left\{\begin{array}{l}
x(t, u)=u+t \frac{\partial H}{\partial p}\left(\frac{\partial \phi}{\partial x}(u)\right) \\
p(t, u)=\frac{\partial \phi}{\partial x}(u) \\
y(t, u)=t\left\{-H\left(\frac{\partial \phi}{\partial x}(u)\right)+\left\langle\frac{\partial \phi}{\partial x}(u), \frac{\partial H}{\partial p}\left(\frac{\partial \phi}{\partial x}(u)\right)\right\rangle\right\}+\phi(u) .
\end{array}\right.
$$

We consider a family of functions $F(t, x, p, q)=\phi(q)+\langle p,(x-q)\rangle-H(p) t$, where $(t, x, p, q) \in \mathbb{R} \times \mathbb{R}^{n} \times\left(\mathbb{R}^{n} \times \mathbb{R}^{n}\right)$ and $\langle$,$\rangle is the canonical inner product on \mathbb{R}^{n}$. We have

$$
\Sigma(F)=\left\{\left(t, q+\frac{\partial H}{\partial p}\left(\frac{\partial \phi}{\partial q}(q)\right) t, \frac{\partial \phi}{\partial q}(q), q\right) \mid(t, q) \in \mathbb{R} \times \mathbb{R}^{n}\right\}
$$

where $\Sigma(F)$ is the set defined by $\frac{\partial F}{\partial p_{i}}=0$ and $\frac{\partial F}{\partial q_{i}}=0$. We now define a map $\Phi_{F}: \Sigma(F) \rightarrow$ $J^{1}\left(\mathbb{R} \times \mathbb{R}^{n}, \mathbb{R}\right)$ by $\Phi_{F}(t, x, p, q)=\left(t, x, F(t, x, p, q), \frac{\partial F}{\partial t}, \frac{\partial F}{\partial x}\right)$. It follows that

$$
\begin{aligned}
\Phi_{F}\left(t, q+\frac{\partial H}{\partial p}\left(\frac{\partial \phi}{\partial q}(q)\right) t, \frac{\partial \phi}{\partial q}(q), q\right) & \\
=\left(t, q+\frac{\partial H}{\partial p}\left(\frac{\partial \phi}{\partial q}(q)\right) t,-H\left(\frac{\partial \phi}{\partial q}(q)\right) t+\left\langle\frac{\partial \phi}{\partial q}(q), \frac{\partial H}{\partial p}\left(\frac{\partial \phi}{\partial q}(q)\right)\right\rangle+\phi(q),\right. & \left.-H\left(\frac{\partial \phi}{\partial q}(q)\right), \frac{\partial \phi}{\partial q}(q)\right) .
\end{aligned}
$$

This shows that the image of the map $\Phi_{F}$ is equal to $L_{\phi, t}$, namely, $F$ is a global generating family of $L_{\phi, t}$.

We refer to the following result of Bardi-Evans [2].

TheOREM 3.2. Assume that the Hamiltonian $H\left(p_{1}, \ldots, p_{n}\right)$ is convex, then

$$
y(t, x) \equiv \inf _{q} \sup _{p}\{\phi(q)+\langle p, x-q\rangle-H(p) t\}
$$

is the unique viscosity solution of $(\mathrm{P})$.

Then we have the following theorem as a corollary of the above theorem.

THEOREM 3.3. Assume that $H$ is uniformly convex and $\phi$ has a minimum. Let $L_{\phi, t}$ be the geometric solution $(\mathrm{S})$ of the Cauchy problem $(\mathrm{P})$. Then

$$
y(t, x) \equiv \min _{y}\left\{y \mid(t, x, y) \in \Pi\left(L_{\phi, t}\right)\right\}
$$

is the unique viscosity solution of $\left(\mathrm{P}^{\prime}\right)$.

Pr o of. Consider the family of functions $F(t, x, p, q)=\phi(q)+\langle p, x-q\rangle-H(p) t$. Since $H(p)$ is uniformly convex, we have

$$
\sup _{p}\{\phi(q)+\langle p, x-q\rangle-H(p) t\}=F \mid \Sigma_{p}(F),
$$


where $\Sigma_{p}(F)=\left\{(t, x, p, q) \mid \frac{\partial F}{\partial p_{i}}=x_{i}-q_{i}-\frac{\partial H}{\partial p_{i}}(p) t=0, i=1, \ldots, n\right\}$. It follows that

$$
\inf _{q} \sup _{p}\{\phi(q)+\langle p, x-q\rangle-H(p) t\}=\inf _{q}\left\{F\left(t, q+\frac{\partial H}{\partial p}(p) t, p, q\right)\right\} .
$$

Since $\phi$ has a minimum, it is equal to

$$
\min _{q}\left\{F\left(t, q+\frac{\partial H}{\partial p}(p) t, p, q\right)\right\}=\min _{q}\left\{F(t, x, p, q) \mid(t, x, p, q) \in \Sigma_{p}(F)\right\} .
$$

On the other hand, we define functions $f_{i}(t, x, p, q)=\frac{\partial F}{\partial p_{i}}=x_{i}-q_{i}-\frac{\partial H}{\partial p_{i}}(p) t(i=$ $1, \ldots, n)$. Since $H(p)$ is uniformly convex, we have $\frac{\partial f_{i}}{\partial p_{j}}\left(t_{0}, x_{0}, p_{0}, q_{0}\right)=-\frac{\partial^{2} H}{\partial p_{i} \partial p_{j}}\left(p_{0}\right) t_{0} \neq 0$, at any point $\left(t_{0}, x_{0}, p_{0}, q_{0}\right)$, so that there exist local smooth functions $g_{i}(t, x, q)(i=$ $1, \ldots, n)$ near $\left(t_{0}, x_{0}, p_{0}, q_{0}\right)$ such that $\Sigma_{p}(F)=\left\{p_{i}=g_{i}(t, x, q)\right\}$. Thus we have

$$
\begin{aligned}
\frac{\partial F \mid \Sigma_{p}(F)}{\partial q_{i}} & =\sum_{j} \frac{\partial F}{\partial p_{j}}(t, x, g(t, x, q), q) \frac{\partial g_{j}}{\partial q_{i}}(t, x, q)+\frac{\partial \phi}{\partial q_{i}}(q)-g_{i}(t, x, q) \\
& =\frac{\partial \phi}{\partial q_{i}}(q)-g_{i}(t, x, q),
\end{aligned}
$$

so that $\Sigma(F)=\left\{(t, x, p, q) \in \Sigma_{p}(F) \mid \frac{\partial F \mid \Sigma_{p}(F)}{\partial q_{i}}=0\right\}$. It follows that

$$
\begin{aligned}
y(t, x) & \equiv \min _{y}\left\{y \mid(t, x, y) \in \Pi\left(L_{\phi, t}\right)\right\}=\min _{(p, q)}\{F(t, x, p, q) \mid(t, x, p, q) \in \Sigma(F)\} \\
& =\min _{q}\left\{F(t, x, p, q) \mid(t, x, p, q) \in \Sigma_{p}(F)\right\} .
\end{aligned}
$$

It is the unique viscosity solution for the Cauchy problem $(\mathrm{P})$.

However, for general (non-convex) Hamiltonian, situations are quite different.

4. Non-convex Hamiltonians in one space variable. In this section we stick to the Cauchy problem of Hamilton-Jacobi equation in one space variable as follows:

$$
\left\{\begin{array}{l}
\frac{\partial y}{\partial t}+H\left(\frac{\partial y}{\partial x}\right)=0 \\
y(0, x)=\phi(x)
\end{array}\right.
$$

where $H$ and $\phi$ are $C^{\infty}$-functions. Since $H(p)$ is not assumed to be uniformly convex (or concave), we cannot use Theorem 3.3, so that the situations should be quite complicated even for the one space variable case.

In this case the geometric solution is given by

$$
L_{\phi, t}=\{(t, x(t, u), y(t, u),-H(p(t, u)), p(t, u)) \mid u \in \mathbb{R}\},
$$

where

$$
\left\{\begin{array}{l}
x(t, u)=u+t H^{\prime}\left(\phi^{\prime}(u)\right) \\
p(t, u)=\phi^{\prime}(u) \\
y(t, u)=t\left\{-H\left(\phi^{\prime}(u)\right)+\phi^{\prime}(u) H^{\prime}\left(\phi^{\prime}(u)\right)\right\}+\phi(u) .
\end{array}\right.
$$

Before the first critical time that characteristics cross in the $(t, x)$-plane, $W_{t}$ is the graph of the viscosity solution $y_{\mathfrak{v}}$. After the characteristics cross, $W_{t}$ becomes singular. Theorem 2.2 describes the generic singularities of $W_{t}$. The first singularity appears in the form of ${ }^{1} A_{3}$. See Figure 1a, where we show the shape of the appearing singularity. 


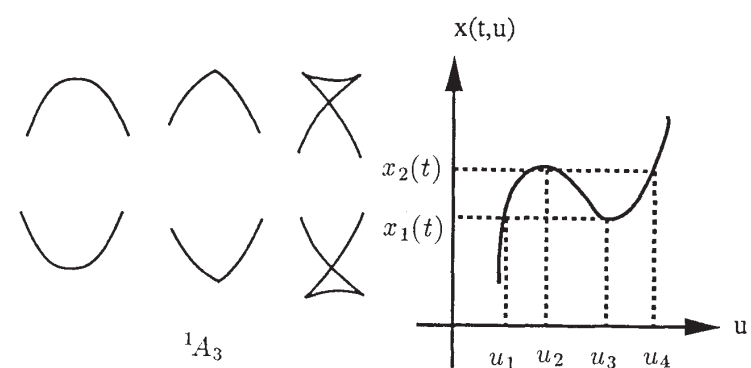

Fig. 1a

Fig. 1b

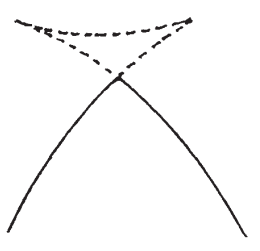

Fig. 1c

By Theorem 2.5, these appear at the convex or the concave points of the Hamiltonian function. Away from the singularity, the viscosity solution is given by $W_{t}$. In ([17], [18]) we have constructed the unique viscosity solution past the first critical time by selecting a single-valued branch of $W_{t}$. Assume that the singularity of type ${ }^{1} A_{3}$ appears at the point $\left(t_{0}, x_{0}, p_{0}\right)$. After the critical time $t_{0}$, the wave front $W_{t}$ is three-valued on an interval $\left(x_{1}(t), x_{2}(t)\right)$; see Figure 1b. Let $y_{i}, i=1,2,3$ be the three branches of $W_{t}$, where $y_{1}$ is defined on a neighborhood of $x_{1}(t)$ and $y_{2}$ on a neighborhood of $x_{2}(t)$. Then $y_{1}, y_{2}$ intersect at one point $\chi(t) \in\left(x_{1}(t), x_{2}(t)\right)$, for $t>t_{0}$. We define the viscosity solution past $t_{0}$ by selecting a continuous single-valued branch of $W_{t}$ as follows:

THEOREM 4.1. There exists an $\varepsilon>0$ such that the function $y_{\mathfrak{v}}(t, x),(t, x) \in\left(t_{0}, t_{0}+\right.$ $\varepsilon) \times\left(x_{1}(t), x_{2}(t)\right)$, defined by

$$
y_{\mathfrak{v}}(t, x)= \begin{cases}y_{1}(t, x), & x \leq \chi(t) \\ y_{3}(t, x), & x \geq \chi(t)\end{cases}
$$

is the viscosity solution of $(\mathrm{P})$ in a neighborhood of $x_{0}$ past the time $t_{0}$.

In view of Theorem 2.5 the viscosity criterion (see Section 3 ) is satisfied across $\chi(t)$ while $y_{\mathfrak{v}}$ defined by $(4.1)$ is a classical solution away from $\chi(t)$. Hence, by the uniqueness of the viscosity solution, (4.1) gives the viscosity solution of $(\mathrm{P})$ past $t_{0}$.

By this construction, we have extended the viscosity solution beyond the first critical time $t_{0}$. According to Theorem 2.5 the shock is generated in a convex or concave domains of $H(p)$, so the viscosity criterion is automatically satisfied. The graph of the viscosity solution past the first critical time is depicted by a full line in Figure 1c, where we assume that $H$ is convex in the neighborhood of the appearing singularity ${ }^{1} A_{3}$. The shock corresponds to the intersection of the two branches and it is called a genuine shock. The genuine shock is defined as the intersection of two incoming characteristics (or waves) and its speed is given by the Rankine-Hugoniot condition

$$
\chi^{\prime}(t)=\frac{H\left(y_{\mathfrak{v}, x}^{+}(t, \chi(t))\right)-H\left(y_{\mathfrak{v}, x}^{-}(t, \chi(t))\right)}{y_{\mathfrak{v}, x}^{+}(t, \chi(t))-y_{\mathfrak{v}, x}^{-}(t, \chi(t))},
$$

where $y_{\mathfrak{v}, x}^{ \pm}=\frac{\partial y_{\mathfrak{v}}^{ \pm}}{\partial x}$ and $\chi^{\prime}(t)=\frac{d \chi}{d t}(t)$. Therefore in order to follow the evolution of the shock we have to study the following questions: 
a) How different branches of the multi-valued graph of $W_{t}$ intersecting at one point bifurcate in time.

b) If the two branches initially defining the shock continue to cross, whether the viscosity criterion is satisfied across the intersection.

The normal forms of the generic bifurcations of different branches of $W_{t}$ are given in Theorem 2.2. We depict these bifurcations in Figure 2.
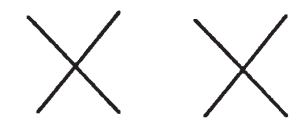

$$
{ }^{0}\left({ }^{0} A_{1}{ }^{0} A_{1}\right)
$$

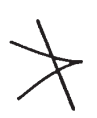

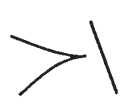

${ }^{1} A_{2}{ }^{0} A_{1}$
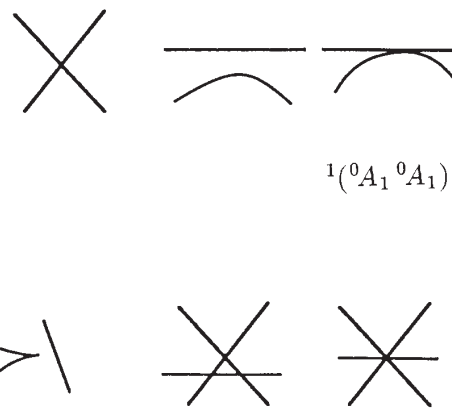

${ }^{0} A_{1}{ }^{0} A_{1}{ }^{0} A_{1}$

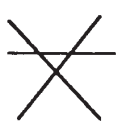

Fig. 2

If the viscosity criterion is satisfied at the time $t_{\alpha}=t_{0}+\varepsilon$, we can choose the correct branch of the graphs of the geometric solutions as viscosity solutions (see Figure 3 ).

We will now investigate how the viscosity criterion can be violated across the intersection of two branches. Assume that a generated shock is defined by two intersecting branches $y^{-}$and $y^{+}$. We denote by $y^{-}$(resp. $y^{+}$) the branch representing the viscosity solution for $x<\chi(t)$ (resp. $x>\chi(t)$ ). If the two branches remain intersected they evolve according to ${ }^{0}\left({ }^{0} A_{1}{ }^{0} A_{1}\right)$. We denote by $\chi(t)$ the intersection of the two branches. In the case when $H(p)$ has only one inflection point Kossioris [17] studied this problem and constructed the viscosity solutions. We consider the general situation here. It is clear that for generic Hamiltonian function $H(p), H$ has only Morse type critical points and no tritangent lines. So we assume that the Hamiltonian has the above properties. By Theorem 2.2, we have the following theorem.

THEOREM 4.2. For a generic initial function $\phi$, if the viscosity criterion is violated at $t_{\alpha}$, then the only following 8 cases may occur:

(1) The normal form is ${ }^{0}\left({ }^{0} A_{1}{ }^{0} A_{1}\right)$ and $\overline{P^{+} P^{-}}$is tangent to the graph of $H(p)$ at only one of the points $P^{+}, P^{-}$and the line is not tangent to the graph at other points between these points.

(2) The normal form is ${ }^{0}\left({ }^{0} A_{1}{ }^{0} A_{1}\right)$ and $\overline{P^{+} P^{-}}$is not tangent to the graph of $H(p)$ at each point $P^{+}, P^{-}$and there exists only one more point between these points at which the above line is tangent to the graph.

(3) The normal form is ${ }^{0}\left({ }^{0} A_{1}{ }^{0} A_{1}\right)$ and $\overline{P^{+} P^{-}}$is tangent to the graph of $H(p)$ at only one of the points $P^{+}, P^{-}$and there exists only one more point between these points at 

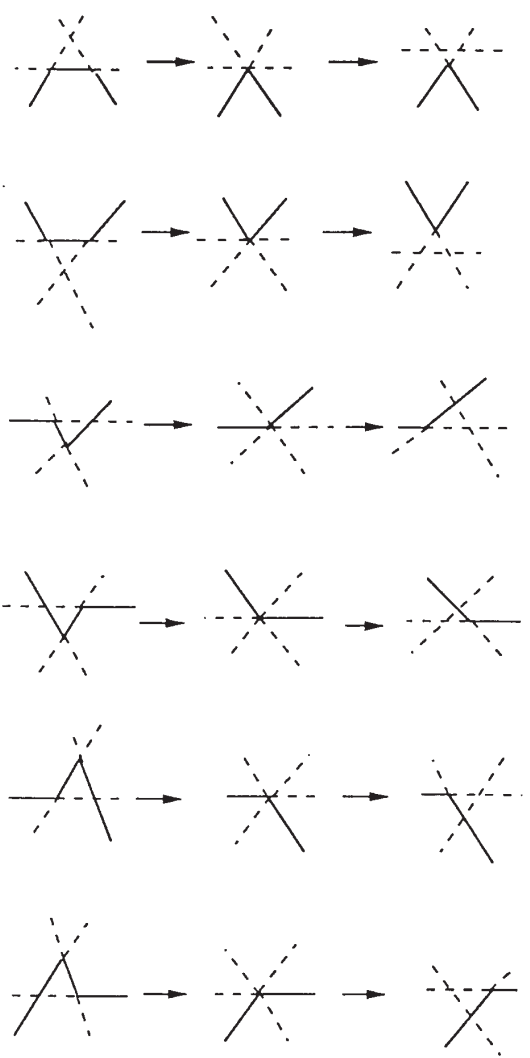

Fig. 3

which the above line is tangent to the graph.

(4) The normal form is ${ }^{0}\left({ }^{0} A_{1}{ }^{0} A_{1}\right)$ and $\overline{P^{+} P^{-}}$is tangent to the graph of $H(p)$ at each point of $P^{+}, P^{-}$.

(5) The normal form is ${ }^{0}\left({ }^{0} A_{1}{ }^{0} A_{1}\right)$ and $\overline{P^{+} P^{-}}$is not tangent to the graph of $H(p)$ at each point $P^{+}, P^{-}$and there exist exactly two other points between these points at which the above line is tangent to the graph.

(6) The normal form is ${ }^{1} A_{2}{ }^{0} A_{1}$ and $\overline{P^{+} P^{-}}$is tangent to the graph of $H(p)$ at only one of the points $\mathrm{P}^{+}, \mathrm{P}^{-}$and the line is not tangent to the graph at other points between these points.

(7) The normal form is ${ }^{0} A_{1}{ }^{0} A_{1}{ }^{0} A_{1}$ and $\overline{P^{+} P^{-}}$is tangent to the graph of $H(p)$ at only one of the points $P^{+}, P^{-}$and is not tangent to the graph at other points between these points.

(8) The normal form is ${ }^{0} A_{1}{ }^{0} A_{1}{ }^{0} A_{1}$ and $\overline{P^{+} P^{-}}$is not tangent to the graph of $H(p)$ at each point $P^{+}, P^{-}$and there exists only one more point between these points at which the above line is tangent to the graph.

Here, $P^{+}=\left(y_{x}^{+}\left(t_{\alpha}, \chi\left(t_{\alpha}\right)\right), H\left(y_{x}^{+}\left(t_{\alpha}, \chi\left(t_{\alpha}\right)\right)\right)\right), P^{-}=\left(y_{x}^{-}\left(t_{\alpha}, \chi\left(t_{\alpha}\right)\right), H\left(y_{x}^{-}\left(t_{\alpha}, \chi\left(t_{\alpha}\right)\right)\right)\right)$ and $\overline{P^{+} P^{-}}$denotes the line through $P^{+}, P^{-}$in the $(p, H(p))$-plane. 
Proof. By Theorem 2.2, we may assume that the first singularities appear in the form of ${ }^{1} A_{3}$. After that the singularities of the graph of the geometric solution bifurcate in the forms of ${ }^{0}\left({ }^{0} A_{1}{ }^{0} A_{1}\right),{ }^{1}\left({ }^{0} A_{1}{ }^{0} A_{1}\right),{ }^{1} A_{2}{ }^{0} A_{1}$ or ${ }^{0} A_{1}{ }^{0} A_{1}{ }^{0} A_{1}$. Since the characteristics in $J^{1}(\mathbb{R} \times \mathbb{R}, \mathbb{R})$ never cross, we can get rid of the case ${ }^{1}\left({ }^{0} A_{1}{ }^{0} A_{1}\right)$.

We already mentioned that the viscosity criterion is satisfied past the first critical time $t_{0}$, so that it is satisfied until the time $t_{\alpha}$ when $\overline{P^{+} P^{-}}$is tangent to the graph of $H(p)$. By the assumptions on the Hamiltonian $H(p)$, we may consider the case where $\overline{P^{+} P^{-}}$is at most a double tangent line for each normal form. We now distinguish each normal form. We write $p^{+}=y_{x}^{+}\left(t_{\alpha}, \chi\left(t_{\alpha}\right)\right)=\phi^{\prime}\left(u_{+}\right)$and $p^{-}=y_{x}^{-}\left(t_{\alpha}, \chi\left(t_{\alpha}\right)\right)=\phi^{\prime}\left(u_{-}\right)$.

(A) ${ }^{0}\left({ }^{0} A_{1}{ }^{0} A_{1}\right)$ : In this case each branch of the graph of geometric solution is a nonsingular curve. We remark that $y^{ \pm}(t, \chi(t))=t\left\{-H\left(\phi^{\prime}\left(u_{ \pm}\right)\right)+\phi^{\prime}\left(u_{ \pm}\right) H^{\prime}\left(\phi\left(u_{ \pm}\right)\right)\right\}+\phi\left(u_{ \pm}\right)$. Since the normal form ${ }^{0}\left({ }^{0} A_{1}{ }^{0} A_{1}\right)$ has trivial bifurcations along the time parameter, the condition $y^{+}(t, \chi(t))=y^{-}(t, \chi(t))$ defines a codimension 0 submanifold in the corresponding jet space, so that we may ignore this condition. We now consider the following conditions which correspond to all possible cases:

(a) $\pm H^{\prime}\left(\phi^{\prime}\left(u_{+}\right)\right)=\frac{H\left(\phi^{\prime}\left(u_{+}\right)\right)-H\left(\phi^{\prime}\left(u_{-}\right)\right)}{\phi^{\prime}\left(u_{+}\right)-\phi^{\prime}\left(u_{-}\right)}$, which defines a submanifold in ${ }_{2} J^{1}(\mathbb{R}, \mathbb{R})$ of codimension 1. Of course, we have to consider the case where

$$
\pm H^{\prime}\left(\phi^{\prime}\left(u_{-}\right)\right)=\frac{H\left(\phi^{\prime}\left(u_{+}\right)\right)-H\left(\phi^{\prime}\left(u_{-}\right)\right)}{\phi^{\prime}\left(u_{+}\right)-\phi^{\prime}\left(u_{-}\right)} ;
$$

however, this case is essentially contained in the above, so that we may ignore such non-essentially different cases in the following arguments.

(b) There exists $u_{0}$ with $u_{0} \neq u_{ \pm}$such that

$$
\pm H^{\prime}\left(\phi^{\prime}\left(u_{0}\right)\right)=\frac{H\left(\phi^{\prime}\left(u_{+}\right)\right)-H\left(\phi^{\prime}\left(u_{-}\right)\right)}{\phi^{\prime}\left(u_{+}\right)-\phi^{\prime}\left(u_{-}\right)} .
$$

This condition defines a submanifold in ${ }_{2} J^{1}(\mathbb{R}, \mathbb{R})$ of codimension 1 .

(c) There exists $u_{0}$ with $u_{0} \neq u_{ \pm}$such that

$$
\pm H^{\prime}\left(\phi^{\prime}\left(u_{+}\right)\right)= \pm H^{\prime}\left(\phi^{\prime}\left(u_{0}\right)\right)=\frac{H\left(\phi^{\prime}\left(u_{+}\right)\right)-H\left(\phi^{\prime}\left(u_{-}\right)\right)}{\phi^{\prime}\left(u_{+}\right)-\phi^{\prime}\left(u_{-}\right)}=\frac{H\left(\phi^{\prime}\left(u_{+}\right)\right)-H\left(\phi^{\prime}\left(u_{0}\right)\right)}{\phi^{\prime}\left(u_{+}\right)-\phi^{\prime}\left(u_{0}\right)} \text {. }
$$

This condition defines a submanifold in ${ }_{3} J^{1}(\mathbb{R}, \mathbb{R})$ of codimension 3 .

(d) $\pm H^{\prime}\left(\phi^{\prime}\left(u_{+}\right)\right)= \pm H^{\prime}\left(\phi^{\prime}\left(u_{-}\right)\right)=\frac{H\left(\phi^{\prime}\left(u_{+}\right)\right)-H\left(\phi^{\prime}\left(u_{-}\right)\right)}{\phi^{\prime}\left(u_{+}\right)-\phi^{\prime}\left(u_{-}\right)}$, which defines a submanifold in ${ }_{2} J^{1}(\mathbb{R}, \mathbb{R})$ of codimension 2 .

(e) There exist $u_{0}, u_{1}$ which are different from $u_{ \pm}$such that

$$
\begin{aligned}
\pm H^{\prime}\left(\phi^{\prime}\left(u_{0}\right)\right) & = \pm H^{\prime}\left(\phi^{\prime}\left(u_{1}\right)\right)=\frac{H\left(\phi^{\prime}\left(u_{+}\right)\right)-H\left(\phi^{\prime}\left(u_{-}\right)\right)}{\phi^{\prime}\left(u_{+}\right)-\phi^{\prime}\left(u_{-}\right)} \\
& =\frac{H\left(\phi^{\prime}\left(u_{+}\right)\right)-H\left(\phi^{\prime}\left(u_{0}\right)\right)}{\phi^{\prime}\left(u_{+}\right)-\phi^{\prime}\left(u_{0}\right)}=\frac{H\left(\phi^{\prime}\left(u_{+}\right)\right)-H\left(\phi^{\prime}\left(u_{1}\right)\right)}{\phi^{\prime}\left(u_{+}\right)-\phi^{\prime}\left(u_{1}\right)} .
\end{aligned}
$$

This condition defines a submanifold of ${ }_{4} J^{1}(\mathbb{R}, \mathbb{R})$ of codimension 4 . Here, ${ }_{r} J^{1}(\mathbb{R}, \mathbb{R})$ is a multi-1-jet space of function germ $\mathbb{R} \rightarrow \mathbb{R}$. Each submanifold in ${ }_{r} J^{1}(\mathbb{R}, \mathbb{R})$ has at most codimension $r$, so that we cannot avoid such conditions by the multi-jet transversality theorem. 
(B) ${ }^{1} A_{2}{ }^{0} A_{1}$ : In this case the normal form ${ }^{1} A_{2}{ }^{0} A_{1}$ bifurcates at the time $t_{\alpha}$, so that we should consider the condition $y^{+}\left(t_{\alpha}, \chi\left(t_{\alpha}\right)\right)=y^{-}\left(t_{\alpha}, \chi\left(t_{\alpha}\right)\right)$ for fixed $t_{\alpha}$. It defines a submanifold in ${ }_{2} J^{1}(\mathbb{R}, \mathbb{R})$ of codimension 1 . By the same arguments as above, we can avoid the conditions (c), (d) and (e). So we may consider the condition (a) or (b). We now show that the condition (a) holds for the normal form ${ }^{1} A_{2}{ }^{0} A_{1}$. On the $(t, x)$-plane, we denote $(t, \chi(t))$ the genuine shocks for $t \leq t_{\alpha}$. Suppose that the point $u_{-}$corresponds to the cusp point at the time $t_{\alpha}$. Then there exists a smooth function $u(t)$ such that $\chi(t)=u(t)+t H^{\prime}\left(\phi^{\prime}(u(t))\right)$ for $t \leq t_{\alpha}$ and $u\left(t_{\alpha}\right)=u_{-}$, where we choose one of the branches of the graph of the geometric solution corresponding to $u_{-}$. It follows that we have

$$
\chi^{\prime}(t)=u^{\prime}(t)\left(1+t H^{\prime \prime}\left(\phi^{\prime}(u(t))\right) \phi^{\prime \prime}(t)\right)+H^{\prime}\left(\phi^{\prime}(u(t))\right)
$$

Since the graph of the geometric solution has a singularity at $t_{\alpha}$, we have $\frac{\partial x}{\partial u}\left(t_{\alpha}, u_{-}\right)=$ $1+t_{\alpha} H^{\prime \prime}\left(\phi^{\prime}\left(u_{-}\right)\right) \phi^{\prime \prime}\left(u_{-}\right)=0$. So we have $\chi^{\prime}\left(t_{\alpha}\right)=\lim _{t \rightarrow t_{\alpha}} \chi^{\prime}(t)=H^{\prime}\left(\phi^{\prime}\left(u_{-}\right)\right)$.

On the other hand, by the Rankine-Hugoniot condition we have

$$
\chi^{\prime}(t)=\frac{H\left(y_{\mathfrak{v}, x}^{+}(t, \chi(t))\right)-H\left(y_{\mathfrak{v}, x}^{-}(t, \chi(t))\right)}{y_{\mathfrak{v}, x}^{+}(t, \chi(t))-y_{\mathfrak{v}, x}^{-}(t, \chi(t))},
$$

for $t \leq t_{\alpha}$. Since $\lim _{t \rightarrow t_{\alpha}} y_{\mathfrak{v}, x}^{ \pm}(t, \chi(t))=\phi^{\prime}\left(u_{ \pm}\right)$, we have $\chi^{\prime}\left(t_{\alpha}\right)=\frac{H\left(\phi^{\prime}\left(u_{+}\right)\right)-H\left(\phi^{\prime}\left(u_{-}\right)\right)}{\phi^{\prime}\left(u_{+}\right)-\phi^{\prime}\left(u_{-}\right)}$, so that we have $H^{\prime}\left(\phi^{\prime}\left(u_{-}\right)\right)=\frac{H\left(\phi^{\prime}\left(u_{+}\right)\right)-H\left(\phi^{\prime}\left(u_{-}\right)\right)}{\phi^{\prime}\left(u_{+}\right)-\phi^{\prime}\left(u_{-}\right)}$. This condition corresponds to the case (a) and we may get rid of the case (b).

(C) ${ }^{0} A_{1}{ }^{0} A_{1}{ }^{0} A_{1}$ : In this case the normal form also bifurcates at the point $t_{\alpha}$, so that we can get rid of the case (c), (d) and (e) by similar reasons to those for the case (B). Since each branch of the normal form is non-singular, the remaining two cases may occur in general. This completes the proof.

We can solve local Riemann problems and construct viscosity solutions for each case in the above theorem. However, we only consider the cases (1) and (6) in this paper. We will give the detailed considerations for all cases elsewhere.

Case (1). We assume that the graph of the viscosity solution at the time $t \leq t_{\alpha}$ is as depicted in Figure 4a.

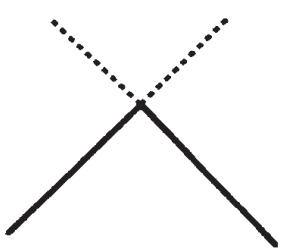

Fig. $4 \mathrm{a}$

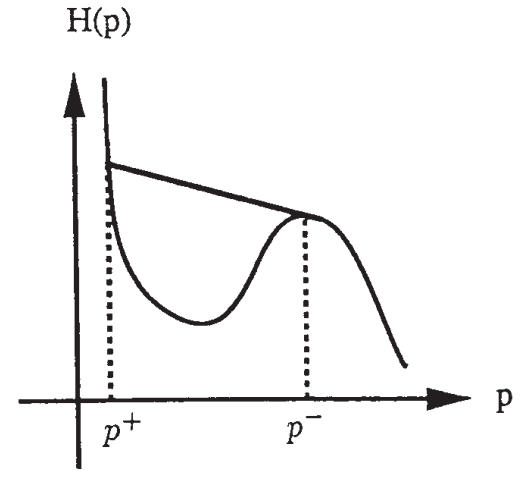

Fig. 4b 
Without the loss of generality, we may assume that $\overline{P^{+} P^{-}}$is tangent to the graph of $H(p)$ at the point $\left(y_{x}^{-}\left(t_{\alpha}, \chi\left(t_{\alpha}\right)\right), H\left(y_{x}^{-}\left(t_{\alpha}, \chi\left(t_{\alpha}\right)\right)\right)\right.$ ) and $H^{\prime \prime}\left(y_{x}^{-}\left(t_{\alpha}, \chi\left(t_{\alpha}\right)\right)\right)<0$ (see Figure $4 \mathrm{~b})$. As we already mentioned, the genuine shocks satisfy the Rankin-Hugoniot condition. So we should construct new characteristics which satisfies both the RankinHugoniot condition and the viscosity criterion. In this case we have

$$
H^{\prime}\left(y_{x}^{-}\left(t_{\alpha}, \chi\left(t_{\alpha}\right)\right)\right)=\frac{H\left(y_{x}^{+}\left(t_{\alpha}, \chi\left(t_{\alpha}\right)\right)\right)-H\left(y_{x}^{-}\left(t_{\alpha}, \chi\left(t_{\alpha}\right)\right)\right)}{y_{x}^{+}\left(t_{\alpha}, \chi\left(t_{\alpha}\right)\right)-y_{x}^{-}\left(t_{\alpha}, \chi\left(t_{\alpha}\right)\right)}=\chi^{\prime}\left(t_{\alpha}\right) .
$$

We now distinguish two cases as follows:

a) If

$$
H^{\prime}\left(y_{x}^{-}(t, \chi(t))\right) \geq \frac{H\left(y_{x}^{+}(t, \chi(t))\right)-H\left(y_{x}^{-}(t, \chi(t))\right)}{y_{x}^{+}(t, \chi(t))-y_{x}^{-}(t, \chi(t))}
$$

for $t_{\alpha} \leq t<t_{\alpha}+\varepsilon$ for sufficiently small $\varepsilon>0$, then we can easily show that the viscosity criterion is satisfied for $t<t_{\alpha}+\varepsilon$. So we can choose single-valued continuous branches of the geometric solution as the viscosity solution.

b) If

$$
H^{\prime}\left(y_{x}^{-}(t, \chi(t))\right)<\frac{H\left(y_{x}^{+}(t, \chi(t))\right)-H\left(y_{x}^{-}(t, \chi(t))\right)}{y_{x}^{+}(t, \chi(t))-y_{x}^{-}(t, \chi(t))}
$$

for $t_{\alpha} \leq t<t_{\alpha}+\varepsilon$ for sufficiently small $\varepsilon>0$, then we can easily show that the viscosity criterion is violated for $t_{\alpha}<t<t_{\alpha}+\varepsilon$, so that a new way to build the solution is required (cf. Figure 5).
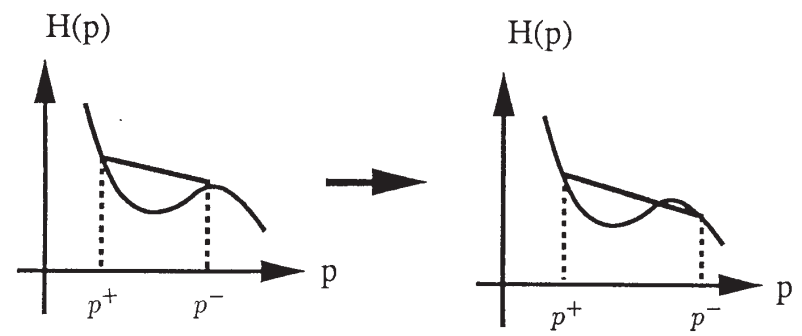

Fig. 5

In this case we can use the techniques in [12] to construct the contact discontinuity shock curve and then obtain new characteristics. Let us consider the relation $H^{\prime}(q)=$ $\frac{H(p)-H(q)}{p-q}$ around $\left(q_{0}, p_{0}\right)$ with $q_{0} \neq p_{0}, H^{\prime}\left(q_{0}\right)=\frac{H\left(p_{0}\right)-H\left(q_{0}\right)}{p_{0}-q_{0}}$ and $H^{\prime \prime}\left(q_{0}\right) \neq 0$. By the implicit function theorem, there exists a smooth function $\psi$ around $p_{0}$ such that the above relation is equivalent to $q=\psi(p)$. We will first construct the contact discontinuity as the solution of the following initial value problem:

$$
\left\{\begin{array}{l}
\chi_{c}^{\prime}(t)=H^{\prime}\left(\psi\left(y_{x}^{+}\left(t, \chi_{c}(t)\right)\right)\right) \\
\chi_{c}\left(t_{\alpha}\right)=\chi\left(t_{\alpha}\right)
\end{array}\right.
$$


The characteristic starting at a point $\left(\tau, \chi_{c}(\tau)\right)$ should satisfy the following:

$$
\left\{\begin{array}{l}
x^{\prime}(t)=H^{\prime}(p(t)) \\
p^{\prime}(t)=0 \\
y^{\prime}(t)=-H(p(t))+p(t) H^{\prime}(p(t))
\end{array}\right.
$$

with initial condition $x(\tau)=\chi_{c}(\tau), y(\tau)=y^{+}\left(\tau, \chi_{c}(\tau)\right)$ and $p(\tau)=\psi\left(y_{x}^{+}\left(\tau, \chi_{c}(\tau)\right)\right)$. So the solution is exactly given as follows:

$$
\left\{\begin{aligned}
\tilde{x}(t)= & \chi_{c}(\tau)+(t-\tau) H^{\prime}\left(\psi\left(y_{x}^{+}\left(\tau, \chi_{c}(\tau)\right)\right)\right) \\
\tilde{p}(t)= & \psi\left(y_{x}^{+}\left(\tau, \chi_{c}(\tau)\right)\right) \\
\tilde{y}(t)= & y^{+}\left(\tau, \chi_{c}(\tau)\right) \\
& +(t-\tau)\left\{-H\left(\psi\left(y_{x}^{+}\left(\tau, \chi_{c}(\tau)\right)\right)\right)+\psi\left(y_{x}^{+}\left(\tau, \chi_{c}(\tau)\right)\right) H^{\prime}\left(\psi\left(y_{x}^{+}\left(\tau, \chi_{c}(\tau)\right)\right)\right)\right\} .
\end{aligned}\right.
$$

By definition of the contact discontinuity, we have

$$
\chi_{c}^{\prime \prime}(t)=H^{\prime \prime}\left(\psi\left(\phi\left(u_{+}(t)\right)\right)\right) \frac{\partial \psi}{\partial p}\left(\phi^{\prime}\left(u_{+}(t)\right)\right) \phi^{\prime \prime}\left(u_{+}(t)\right) u_{+}^{\prime}(t)
$$

where $\chi_{c}(t)=u_{+}(t)+t H^{\prime}\left(\phi\left(u_{+}(t)\right)\right)$. Since $\frac{\partial \psi}{\partial p}=\frac{H^{\prime}(p)-H^{\prime}(q)}{H^{\prime \prime}(q)(p-q)}$, we have

$$
\chi_{c}^{\prime \prime}(t)=\frac{H^{\prime}\left(\phi^{\prime}\left(u_{+}(t)\right)\right)-H^{\prime}\left(\psi\left(\phi^{\prime}\left(u_{+}(t)\right)\right)\right)}{\phi^{\prime}\left(u_{+}(t)\right)-\psi\left(\phi^{\prime}\left(u_{+}(t)\right)\right)} \phi^{\prime \prime}\left(u_{+}(t)\right) u_{+}^{\prime}(t) .
$$

We also have

$$
\chi^{\prime}(t)=u_{+}^{\prime}(t)\left\{1+t H^{\prime \prime}\left(\phi^{\prime}\left(u_{+}(t)\right)\right) \phi^{\prime \prime}\left(u_{+}(t)\right)\right\}+H^{\prime}\left(\phi^{\prime}\left(u_{+}(t)\right)\right) .
$$

It follows that

$$
\chi_{c}^{\prime \prime}(t)=-\frac{\left(H^{\prime}\left(\phi^{\prime}\left(u_{+}(t)\right)\right)-H^{\prime}\left(\psi\left(\phi^{\prime}\left(u_{+}(t)\right)\right)\right)\right)^{2}}{\phi^{\prime}\left(u_{+}(t)\right)-\psi\left(\phi^{\prime}\left(u_{+}(t)\right)\right)} \frac{\phi^{\prime \prime}\left(u_{+}(t)\right)}{1+t H^{\prime \prime}\left(\phi^{\prime}\left(u_{+}(t)\right)\right) \phi^{\prime \prime}\left(u_{+}(t)\right)} .
$$

Since

$$
\frac{\partial x}{\partial u}\left(t, u_{+}(t)\right)=1+t H^{\prime \prime}\left(\phi^{\prime}\left(u_{+}(t)\right)\right) \phi^{\prime \prime}\left(u_{+}(t)\right),
$$

we may assume that $1+t H^{\prime \prime}\left(\phi^{\prime}\left(u_{+}(t)\right)\right) \phi^{\prime \prime}\left(u_{+}(t)\right)>0$. So $\chi_{c}(t)$ is convex if and only if $\phi^{\prime \prime}\left(u_{+}(t)\right)>0$. We suppose that $\phi^{\prime \prime}\left(u_{+}(t)\right) \leq 0$ and denote $\chi_{c}(t)=u_{+}(t)+t H^{\prime}\left(\phi\left(u_{+}(t)\right)\right)$ $=u_{-}(t)+t H^{\prime}\left(\phi\left(u_{-}(t)\right)\right)$, where $u_{-}(t)$ (resp. $\left.u_{+}(t)\right)$ is the point corresponding to the characteristic from the right (resp. left) side of $\left(t, \chi_{c}(t)\right)$. We distinguish two cases as follows:

b-1) If $\phi^{\prime \prime}\left(u_{-}(t)\right)>0$, then $\phi^{\prime}$ is monotone. Since $u_{-}^{\prime}(t)<0, \phi_{-}^{\prime}\left(u_{-}(t)\right)$ moves to the left direction, so that the viscosity criterion is satisfied across $\chi$.

b-2) If $\phi^{\prime \prime}\left(u_{-}(t)\right)<0$ and the viscosity criterion is violated across $\chi$ for $t>t_{\alpha}$, then $1+t H^{\prime \prime}\left(\phi^{\prime}\left(u_{-}(t)\right)\right) \phi^{\prime \prime}\left(u_{-}(t)\right)>0$ near $t_{\alpha}$. Differentiate the equality $\chi_{c}(t)=u_{-}(t)+$ $t H^{\prime}\left(\phi\left(u_{-}(t)\right)\right)$ with respect to $t$ to obtain

$$
\chi^{\prime}(t)-H^{\prime}\left(\phi^{\prime}\left(u_{-}(t)\right)\right)=\left\{1+t H^{\prime \prime}\left(\phi^{\prime}\left(u_{-}(t)\right)\right) \phi^{\prime \prime}\left(u_{-}(t)\right)\right\} u_{-}^{\prime}(t) .
$$

Since

$$
\chi^{\prime}(t)=\frac{H\left(\phi^{\prime}\left(u_{+}(t)\right)\right)-H\left(\phi^{\prime}\left(u_{-}(t)\right)\right)}{\phi^{\prime}\left(u_{+}(t)\right)-\phi^{\prime}\left(u_{-}(t)\right)}>H^{\prime}\left(\phi^{\prime}\left(u_{-}(t)\right)\right),
$$

we have $u_{-}^{\prime}(t)>0$, so that $u_{-}(t)$ is increasing, which is a contradiction. 
Hence, if the viscosity criterion is violated for $t>t_{\alpha}$, the contact discontinuity curve $\chi$ is convex and the viscosity solution can be constructed.

We draw a picture which illustrates the situation in Figure 6.

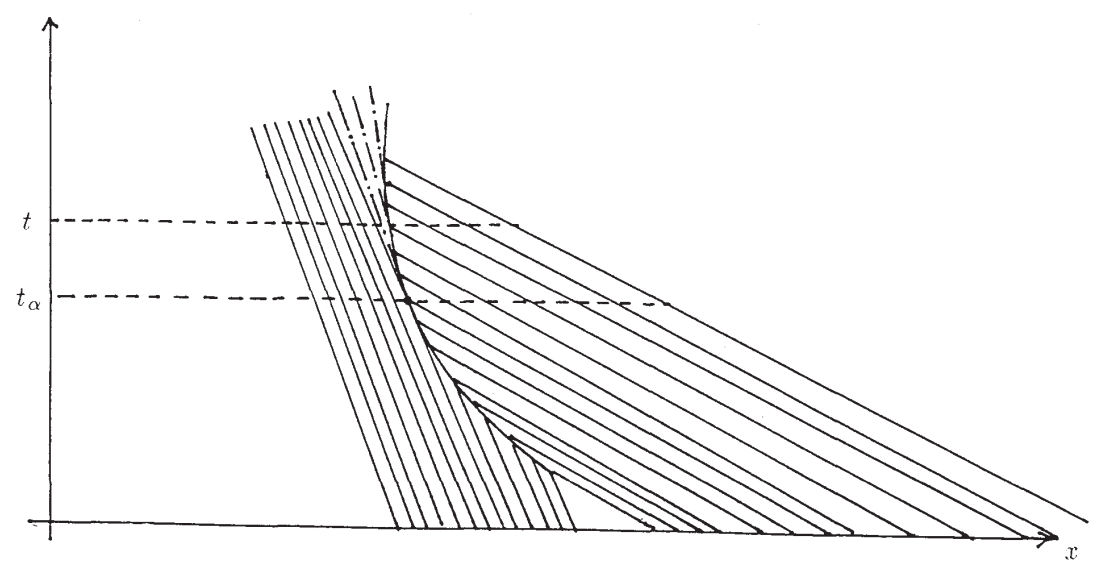

Fig. 6

Figure 7 shows the graph of the viscosity solution for $t>t_{\alpha}$ and the shock curve around $t_{\alpha}$.
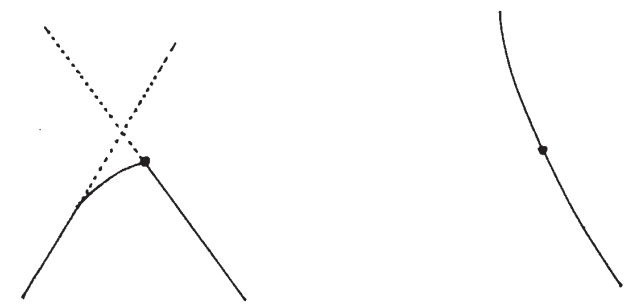

Fig. 7

Case (6). The bifurcations of the graphs of the geometric solution at the time $t_{\alpha}$ are depicted in Figure 8.
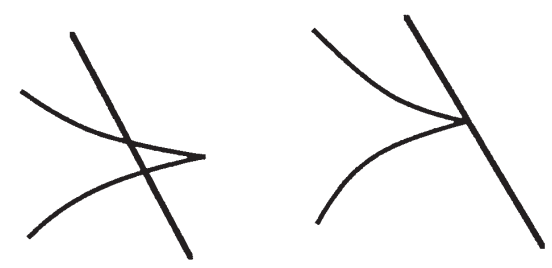

Fig. 8

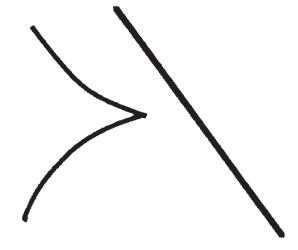


We use the same notation as the case $(1)$. Since $u_{-}\left(t_{\alpha}\right)$ corresponds to the cusp point, we have $1+t_{\alpha} H^{\prime \prime}\left(\phi\left(u_{-}\left(t_{\alpha}\right)\right)\right) \phi^{\prime \prime}\left(u_{-}\left(t_{\alpha}\right)\right)=0$. Let $(t, \sigma(t))$ be the locus of the cusps, where we denote $\sigma(t)=\sigma_{-}(t)+t H^{\prime}\left(\phi^{\prime}\left(\sigma_{-}(t)\right)\right)$ as the family of characteristics coming from the left side, so that we have $1+t H^{\prime \prime}\left(\phi^{\prime}\left(\sigma_{-}(t)\right)\right) \phi^{\prime \prime}\left(\sigma_{-}(t)\right)=0$ and $\sigma_{-}^{\prime}(t)<0$. It follows that $H^{\prime \prime}\left(\phi^{\prime}\left(\sigma_{-}(t)\right)\right) \phi^{\prime \prime}\left(\sigma_{-}(t)\right)<0$ and $\sigma^{\prime}(t)=H^{\prime}\left(\phi^{\prime}\left(\sigma_{-}^{\prime}(t)\right)\right)$. Differentiating the equation again, we get $\sigma^{\prime \prime}(t)=H^{\prime \prime}\left(\phi^{\prime}\left(\sigma_{-}(t)\right)\right) \phi^{\prime \prime}\left(\sigma_{-}(t)\right) \sigma_{-}^{\prime}(t)>0$. Therefore $(t, \sigma(t))$ is strictly convex. We denote $\chi(t)=u_{-}(t)+t H^{\prime}\left(\phi^{\prime}\left(u_{-}(t)\right)\right)=u_{+}(t)+t H^{\prime}\left(\phi^{\prime}\left(u_{+}(t)\right)\right)$ for $t \leq t_{\alpha}$, then we have $u_{-}\left(t_{\alpha}\right)=\sigma_{-}\left(t_{\alpha}\right)$, so that $\sigma^{\prime}\left(t_{\alpha}\right)=H^{\prime}\left(\phi^{\prime}\left(\sigma_{-}\left(t_{\alpha}\right)\right)\right)=H^{\prime}\left(\phi\left(u_{-}\left(t_{\alpha}\right)\right)\right)=\chi^{\prime}\left(t_{\alpha}\right)$ by the proof of Theorem 4.2. We also construct the contact discontinuity $\left(t, \chi_{c}(t)\right)$ exactly as in the case (1). We need to examine the following two subcases.

a) Assume that $\sigma(t) \geq \chi_{c}(t)$ for $t \geq t_{\alpha}$. Since both $\chi_{c}$ and $\sigma$ are convex near $t_{\alpha}$, we have that $\sigma^{\prime \prime}\left(t_{\alpha}\right)>\chi^{\prime \prime}\left(t_{\alpha}\right)$.

On the other hand, we have

$$
\begin{gathered}
y\left(t, \sigma_{ \pm}(t)\right)=t\left\{-H\left(\phi^{\prime}\left(\sigma_{ \pm}(t)\right)\right)+\phi^{\prime}\left(\sigma_{ \pm}(t)\right) H^{\prime}\left(\phi^{\prime}\left(\sigma_{ \pm}(t)\right)\right)\right\}+\phi\left(\sigma_{ \pm}(t)\right), \\
\frac{d y}{d t}\left(t, \sigma_{+}(t)\right)=-H\left(\phi^{\prime}\left(\sigma_{+}(t)\right)\right)+\phi^{\prime}\left(\sigma_{+}(t)\right) H^{\prime}\left(\phi^{\prime}\left(\sigma_{+}(t)\right)\right) \\
+\phi^{\prime}\left(\sigma_{+}(t)\right) \sigma_{+}^{\prime}(t)\left\{1+t H^{\prime \prime}\left(\phi^{\prime}\left(\sigma_{+}(t)\right)\right) \phi^{\prime \prime}\left(\sigma_{+}(t)\right)\right\}
\end{gathered}
$$

and

$$
\frac{d y}{d t}\left(t, \sigma_{-}(t)\right)=-H\left(\phi^{\prime}\left(\sigma_{-}(t)\right)\right)+\phi^{\prime}\left(\sigma_{-}(t)\right) H^{\prime}\left(\phi^{\prime}\left(\sigma_{-}(t)\right)\right) .
$$

Let $A(t)=y\left(t, \sigma_{+}(t)\right)-y\left(t, \sigma_{-}(t)\right)$ for $t \geq t_{\alpha}$.

Differentiating the equality $\sigma_{-}(t)+t H^{\prime}\left(\phi^{\prime}\left(\sigma_{-}(t)\right)\right)=\sigma_{+}(t)+t H^{\prime}\left(\phi^{\prime}\left(\sigma_{+}(t)\right)\right)$ with respect to $t$, we get

$$
\sigma_{+}^{\prime}(t)\left\{1+t H^{\prime \prime}\left(\phi^{\prime}\left(\sigma_{+}(t)\right)\right) \phi^{\prime \prime}\left(\sigma_{+}(t)\right)\right\}=H^{\prime}\left(\phi^{\prime}\left(\sigma_{-}(t)\right)\right)-H^{\prime}\left(\phi^{\prime}\left(\sigma_{+}(t)\right)\right) .
$$

It follows that

$$
A^{\prime}(t)=H^{\prime}\left(\phi\left(\sigma_{-}(t)\right)\right)\left\{\phi^{\prime}\left(\sigma_{+}(t)\right)-\phi^{\prime}\left(\sigma_{-}(t)\right)\right\}-\left(H\left(\phi^{\prime}\left(\sigma_{+}(t)\right)\right)-H\left(\phi^{\prime}\left(\sigma_{-}(t)\right)\right)\right) .
$$

Furthermore, we have

$$
\begin{aligned}
A^{\prime \prime}(t)= & \left(H^{\prime}\left(\phi^{\prime}\left(\sigma_{-}(t)\right)\right)-H^{\prime}\left(\phi^{\prime}\left(\sigma_{+}(t)\right)\right)\right) \phi^{\prime \prime}\left(\sigma_{+}(t)\right) \sigma_{+}^{\prime}(t) \\
& +H^{\prime \prime}\left(\phi^{\prime}\left(\sigma_{-}(t)\right)\right) \phi^{\prime \prime}\left(\sigma_{-}(t)\right)\left\{\phi^{\prime}\left(\sigma_{+}(t)\right)-\phi^{\prime}\left(\sigma_{-}(t)\right)\right\} \sigma_{-}^{\prime}(t) .
\end{aligned}
$$

Since $\sigma_{+}^{\prime}(t)=\frac{H^{\prime}\left(\phi^{\prime}\left(\sigma_{-}(t)\right)\right)-H^{\prime}\left(\phi^{\prime}\left(\sigma_{+}(t)\right)\right)}{1+t H^{\prime \prime}\left(\phi^{\prime}\left(\sigma_{+}(t)\right)\right) \phi^{\prime \prime}\left(\sigma_{+}(t)\right)}$ and $\sigma^{\prime \prime}(t)=H^{\prime \prime}\left(\phi^{\prime}\left(\sigma_{-}(t)\right)\right) \phi^{\prime \prime}\left(\sigma_{-}(t)\right) \sigma_{-}^{\prime}(t)$, we have

$$
A^{\prime \prime}(t)=\frac{\left(H^{\prime}\left(\phi^{\prime}\left(\sigma_{-}(t)\right)\right)-H^{\prime}\left(\phi^{\prime}\left(\sigma_{+}(t)\right)\right)\right)^{2}}{1+t H^{\prime \prime}\left(\phi^{\prime}\left(\sigma_{+}(t)\right)\right) \phi^{\prime \prime}\left(\sigma_{+}(t)\right)} \phi^{\prime \prime}\left(\sigma_{+}(t)\right)+\sigma^{\prime \prime}(t)\left\{\phi^{\prime}\left(\sigma_{+}(t)\right)-\phi^{\prime}\left(\sigma_{-}(t)\right)\right\} .
$$

On the other hand, as we already calculated in the case (1),

$$
\chi_{c}^{\prime \prime}(t)=-\frac{\left(H^{\prime}\left(\phi^{\prime}\left(u_{+}(t)\right)\right)-H^{\prime}\left(\psi\left(\phi^{\prime}\left(u_{+}(t)\right)\right)\right)\right)^{2}}{\phi^{\prime}\left(u_{+}(t)\right)-\psi\left(\phi^{\prime}\left(u_{+}(t)\right)\right)} \frac{\phi^{\prime \prime}\left(u_{+}(t)\right)}{1+t H^{\prime \prime}\left(\phi^{\prime}\left(u_{+}(t)\right)\right) \phi^{\prime \prime}\left(u_{+}(t)\right)} .
$$

At the point $t=t_{\alpha}$, we have $u_{ \pm}\left(t_{\alpha}\right)=\sigma_{ \pm}\left(t_{\alpha}\right)$ and $\psi\left(\phi^{\prime}\left(\sigma_{+}\left(t_{\alpha}\right)\right)\right)=\phi^{\prime}\left(\sigma_{-}\left(t_{\alpha}\right)\right)$, so that

$$
\chi_{c}^{\prime \prime}\left(t_{\alpha}\right)=-\frac{\left(H^{\prime}\left(\phi^{\prime}\left(u_{+}\left(t_{\alpha}\right)\right)\right)-H^{\prime}\left(\psi\left(\phi^{\prime}\left(u_{+}\left(t_{\alpha}\right)\right)\right)\right)^{2}\right.}{\phi^{\prime}\left(u_{+}\left(t_{\alpha}\right)\right)-\psi\left(\phi^{\prime}\left(u_{+}\left(t_{\alpha}\right)\right)\right)} \frac{\phi^{\prime \prime}\left(u_{+}\left(t_{\alpha}\right)\right)}{1+t_{\alpha} H^{\prime \prime}\left(\phi^{\prime}\left(u_{+}\left(t_{\alpha}\right)\right)\right) \phi^{\prime \prime}\left(u_{+}\left(t_{\alpha}\right)\right)} \text {. }
$$


Thus, we have

$$
A^{\prime \prime}\left(t_{\alpha}\right)=\left(\sigma^{\prime \prime}\left(t_{\alpha}\right)-\chi_{c}^{\prime \prime}\left(t_{\alpha}\right)\right)\left(\phi^{\prime}\left(\sigma_{+}\left(t_{\alpha}\right)\right)-\phi^{\prime}\left(\sigma_{-}\left(t_{\alpha}\right)\right)\right) .
$$

Since $\sigma^{\prime \prime}\left(t_{\alpha}\right)>\chi_{c}^{\prime \prime}\left(t_{\alpha}\right)$ and $\phi^{\prime}\left(\sigma_{+}\left(t_{\alpha}\right)\right)<\phi^{\prime}\left(\sigma_{-}\left(t_{\alpha}\right)\right)$, we have $A^{\prime \prime}\left(t_{\alpha}\right)<0$. This means that $A^{\prime}(t)<0$ near $t_{\alpha}$, so $y\left(t, \sigma_{+}(t)\right)<y\left(t, \sigma_{-}(t)\right)$.

We also consider

$$
y_{+}(t)=t\left\{-H\left(\phi^{\prime}\left(u_{+}(t)\right)\right)+\phi^{\prime}\left(u_{+}(t)\right) H^{\prime}\left(\phi^{\prime}\left(u_{+}(t)\right)\right)\right\}+\phi\left(u_{+}(t)\right)
$$

and

$$
y_{\alpha}(t)=t\left\{-H\left(\phi^{\prime}\left(u_{\alpha}\right)\right)+\phi^{\prime}\left(u_{\alpha}\right) H^{\prime}\left(\phi^{\prime}\left(u_{\alpha}\right)\right)\right\}+\phi\left(u_{\alpha}\right),
$$

where $u_{\alpha}=\sigma_{-}\left(t_{\alpha}\right), x\left(t, u_{\alpha}\right)=u_{\alpha}+t H^{\prime}\left(\phi^{\prime}\left(u_{\alpha}\right)\right)=x\left(t, u_{+}(t)\right)=u_{+}(t)+t H^{\prime}\left(\phi^{\prime}\left(u_{+}(t)\right)\right)$. Differentiating the last equality, we get

$$
H^{\prime}\left(\phi^{\prime}\left(u_{\alpha}\right)\right)=u_{+}^{\prime}(t)+H^{\prime}\left(\phi^{\prime}\left(u_{+}(t)\right)\right)+t H^{\prime \prime}\left(\phi^{\prime}\left(u_{+}(t)\right)\right) \phi^{\prime \prime}\left(u_{+}(t)\right) u_{+}^{\prime}(t) .
$$

Then

$$
\begin{aligned}
y_{+}^{\prime}(t)= & -H\left(\phi^{\prime}\left(u_{+}(t)\right)\right)+\phi^{\prime}\left(u_{+}(t)\right) H^{\prime}\left(\phi^{\prime}\left(u_{+}(t)\right)\right) \\
& +t H^{\prime \prime}\left(\phi^{\prime}\left(u_{+}(t)\right)\right) \phi^{\prime}\left(u_{+}(t)\right) \phi^{\prime \prime}\left(u_{+}(t)\right) u_{+}^{\prime}(t)+\phi^{\prime}\left(u_{+}(t)\right) u_{+}^{\prime}(t) \\
= & -H\left(\phi^{\prime}\left(u_{+}(t)\right)\right)+\phi^{\prime}\left(u_{+}(t)\right) H^{\prime}\left(\phi^{\prime}\left(u_{\alpha}\right)\right) .
\end{aligned}
$$

So we obtain

$$
\frac{d}{d t}\left(y_{\alpha}(t)-y_{+}(t)\right)=H\left(\phi^{\prime}\left(u_{+}(t)\right)\right)-H\left(\phi^{\prime}\left(u_{\alpha}\right)\right)-H^{\prime}\left(\phi^{\prime}\left(u_{\alpha}\right)\right)\left(\phi^{\prime}\left(u_{+}(t)\right)-\phi^{\prime}\left(u_{\alpha}\right)\right) .
$$

Since $\phi^{\prime}\left(u_{+}(t)\right)<\phi^{\prime}\left(u_{\alpha}\right)$ and $\phi^{\prime}\left(u_{\alpha}\right)$ is in the convex region of $H(p)$, we have

$$
H^{\prime}\left(\phi^{\prime}\left(u_{\alpha}\right)\right)<\frac{H\left(\phi^{\prime}\left(u_{+}(t)\right)\right)-H\left(\phi^{\prime}\left(u_{\alpha}\right)\right)}{\phi^{\prime}\left(u_{+}(t)\right)-\phi^{\prime}\left(u_{\alpha}\right)},
$$

so that we have $\frac{d}{d t}\left(y_{\alpha}(t)-y_{+}(t)\right)<0$. This means that $y_{\alpha}(t)<y_{+}(t)$ for $t>t_{\alpha}$.

This shows that the two branches of the multi-valued graph have intersection for $t>t-\alpha$. This contradicts the assumption that the singularity is ${ }^{1} A_{2}{ }^{0} A_{1}$.

b) Here we assume that $\sigma(t)<\chi_{c}(t)$ for $t>t_{\alpha}$. In this subcase two shocks bifurcate from the point $\left(t_{\alpha}, x_{\alpha}\right)$ (see Figure 9 ).

The left one is a new shock given by the intersection of the original characteristic from the left side and the new characteristic from the contact discontinuity (i.e., the rarefaction waves).

By definition, we have

$$
\begin{aligned}
\tilde{y}_{\alpha}(t)= & \left(t-t_{\alpha}\right)\left\{-H\left(\psi\left(y_{x}^{+}\left(t_{\alpha}, \chi_{c}\left(t_{\alpha}\right)\right)\right)\right)+\psi\left(y_{x}^{+}\left(t_{\alpha}, \chi_{c}\left(t_{\alpha}\right)\right)\right) H^{\prime \prime}\left(\psi\left(y_{x}^{+}\left(t_{\alpha}, \chi_{c}\left(t_{\alpha}\right)\right)\right)\right)\right\} \\
& +y^{+}\left(t_{\alpha}, \chi\left(t_{\alpha}\right)\right)
\end{aligned}
$$

and

$$
y_{-}(t)=t\left\{-H\left(\phi^{\prime}\left(u_{-}(t)\right)\right)+\phi^{\prime}\left(u_{-}(t)\right) H^{\prime}\left(\phi\left(u_{-}(t)\right)\right)\right\}+\phi\left(u_{-}(t)\right),
$$

where $u_{\alpha}+t H^{\prime}\left(\phi^{\prime}\left(u_{\alpha}\right)\right)=x\left(t, u_{-}(t)\right)=u_{-}(t)+t H^{\prime}\left(\phi^{\prime}(t)\right)$.

Differentiating the last equality, we get

$$
u_{-}^{\prime}(t)+H^{\prime}\left(\phi^{\prime}\left(u_{-}(t)\right)\right)+t H^{\prime \prime}\left(\phi^{\prime}\left(u_{-}(t)\right)\right) \phi^{\prime \prime}\left(u_{-}(t)\right) u_{-}^{\prime}(t)=H^{\prime}\left(\phi^{\prime}\left(u_{\alpha}\right)\right) \text {. }
$$




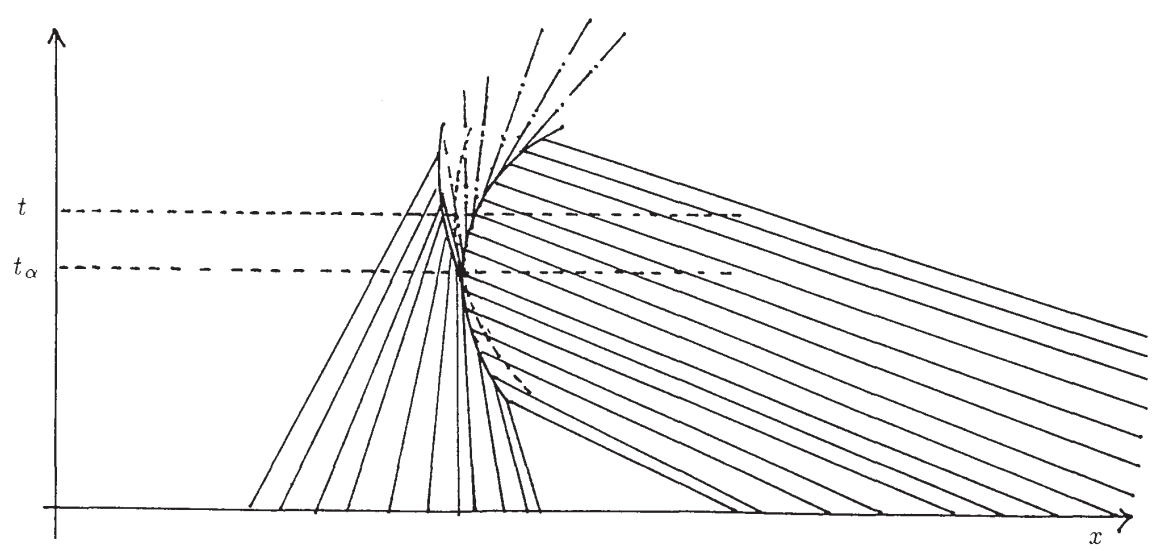

Fig. 9

Then

$$
\begin{aligned}
y_{-}^{\prime}(t)= & -H\left(\phi^{\prime}\left(u_{-}(t)\right)\right)+\phi^{\prime}\left(u_{-}(t)\right) H^{\prime}\left(\phi\left(u_{-}(t)\right)\right) \\
& +t H^{\prime \prime}\left(\phi^{\prime}\left(u_{-}(t)\right)\right) \phi^{\prime}\left(u_{-}(t)\right) \phi^{\prime \prime}\left(u_{-}(t)\right) u_{-}^{\prime}(t)+\phi^{\prime}\left(u_{-}(t)\right) u_{-}^{\prime}(t) \\
= & -H\left(\phi^{\prime}\left(u_{-}(t)\right)\right)+\phi^{\prime}\left(u_{-}(t)\right) H^{\prime}\left(\phi\left(u_{\alpha}\right)\right)
\end{aligned}
$$

So we obtain

$$
\begin{aligned}
\frac{d}{d t}\left(\tilde{y}_{\alpha}(t)-y_{-}(t)\right)= & H\left(\phi^{\prime}\left(u_{-}(t)\right)\right)-H\left(\phi^{\prime}\left(u_{\alpha}\right)\right) \\
& -H^{\prime}\left(\phi^{\prime}\left(u_{\alpha}\right)\right)\left(\phi^{\prime}\left(u_{-}(t)\right)-\phi^{\prime}\left(u_{\alpha}\right)\right) .
\end{aligned}
$$

Since $u_{-}(t)<u_{\alpha}$ and both of $\phi^{\prime}\left(u_{-}(t)\right), \phi^{\prime}\left(u_{\alpha}\right)$ are in the convex region of $H(p)$, we have $\phi^{\prime}\left(u_{-}(t)\right)>\phi^{\prime}\left(u_{\alpha}\right)$ and

$$
H^{\prime}\left(\phi^{\prime}\left(u_{\alpha}\right)\right)>\frac{H\left(\phi^{\prime}\left(u_{-}(t)\right)\right)-H\left(\phi^{\prime}\left(u_{\alpha}\right)\right)}{\phi^{\prime}\left(u_{-}(t)\right)-\phi^{\prime}\left(u_{\alpha}\right)},
$$

so that we obtain $\frac{d}{d t}\left(\tilde{y}_{\alpha}(t)-y_{-}(t)\right)<0$. Since $\tilde{y}_{\alpha}\left(t_{\alpha}\right)=y_{-}\left(t_{\alpha}\right)$, the last inequality means that $\tilde{y}_{\alpha}(t)<y_{-}(t)$ for $t>t_{\alpha}$.

We also consider

$$
\begin{aligned}
\tilde{y}_{\alpha}(t, \sigma(t))= & (t-\tau(t))\left\{-H\left(\psi\left(y_{x}^{+}\left(\tau(t), \chi_{c}(\tau(t))\right)\right)\right)\right. \\
& \left.+\psi\left(y_{x}^{+}\left(\tau(t), \chi_{c}(\tau(t))\right)\right) H^{\prime \prime}\left(\psi\left(y_{x}^{+}\left(\tau(t), \chi_{c}(\tau(t))\right)\right)\right)\right\}+y_{x}^{+}\left(\tau(t), \chi_{c}(\tau(t))\right)
\end{aligned}
$$

and

$$
y_{-}(t, \sigma(t))=t\left\{-H\left(\phi^{\prime}\left(\sigma_{-}(t)\right)\right)+\phi^{\prime}\left(\sigma_{-}(t)\right) H^{\prime}\left(\phi\left(\sigma_{-}(t)\right)\right)\right\}+\phi\left(\sigma_{-}(t)\right)
$$

where

$$
\begin{aligned}
& y^{+}\left(\tau(t), \chi_{c}(\tau(t))\right)=\tau(t)\left\{-H\left(\phi^{\prime}(\tau(t))\right)+\phi^{\prime}(\tau(t)) H^{\prime}(\phi(\tau(t)))\right\}+\phi(\tau(t)), \\
& y_{x}^{+}\left(\tau(t), \chi_{c}(\tau(t))\right)=\phi^{\prime}\left(u_{+}(\tau(t))\right)
\end{aligned}
$$

and

$$
\sigma(t)=\sigma_{-}(t)+t H^{\prime}\left(\phi^{\prime}\left(\sigma_{-}(t)\right)\right)=\chi_{c}(\tau(t))+(t-\tau(t)) H^{\prime}\left(\psi\left(\phi^{\prime}\left(u_{+}(t)\right)\right)\right)
$$


Since $\chi_{c}(\tau)=H^{\prime}\left(\psi\left(\phi^{\prime}\left(u_{+}(\tau)\right)\right)\right)$ and $1+t H^{\prime \prime}\left(\phi^{\prime}\left(\sigma_{-}(t)\right)\right) \phi^{\prime \prime}\left(\sigma_{-}(t)\right)=0$, differentiating the above equality, we get

$$
\begin{aligned}
H^{\prime}\left(\phi^{\prime}\left(\sigma_{-}(t)\right)\right)= & H^{\prime}\left(\psi\left(\phi^{\prime}\left(u_{+}(\tau(t))\right)\right)\right) \\
& +(t-\tau(t)) H^{\prime \prime}\left(\psi\left(\phi\left(u_{+}(\tau(t))\right)\right)\right) \frac{d \psi}{d p} \phi^{\prime}\left(u_{+}(\tau(t))\right) u_{+}^{\prime}(\tau(t)) \tau^{\prime}(t) .
\end{aligned}
$$

Then

$$
\begin{aligned}
\tilde{y}^{\prime}(t, \sigma(t))= & \tau^{\prime}(t)\left\{-H\left(\phi^{\prime}\left(u_{+}(\tau(t))\right)\right)+\phi^{\prime}\left(u_{+}(\tau(t))\right) H^{\prime}\left(\phi^{\prime}\left(u_{+}(\tau(t))\right)\right)\right\} \\
& +\tau(t) \phi^{\prime}\left(u_{+} \tau(t)\right) H^{\prime \prime}\left(\phi^{\prime}\left(u_{+}(\tau(t))\right)\right) \phi^{\prime \prime}\left(u_{+}(\tau(t))\right) u_{+}^{\prime}(\tau(t)) \tau^{\prime}(t) \\
& +\phi^{\prime}\left(u_{+}(\tau(t))\right) u_{+}^{\prime}(\tau(t)) \tau^{\prime}(t) \\
& +\left(1-\tau^{\prime}(t)\right)\left\{-H\left(\psi\left(\phi^{\prime}\left(u_{+}(\tau(t))\right)\right)\right)+\psi\left(\phi^{\prime}\left(u_{+}(\tau(t))\right) H^{\prime}\left(\psi\left(\phi^{\prime}\left(u_{+}(\tau(t))\right)\right)\right)\right)\right\} \\
& +(t-\tau(t)) \psi\left(\phi^{\prime}\left(u_{+}(\tau(t))\right)\right) H^{\prime \prime}\left(\psi\left(\phi^{\prime}\left(u_{+}(\tau(t))\right)\right)\right) \frac{d \psi}{d p} \phi^{\prime \prime}\left(u_{+}(\tau(t))\right) \tau^{\prime}(t) \\
= & \tau^{\prime}(t)\left\{H\left(\psi\left(\phi^{\prime}\left(u_{+}(\tau(t))\right)\right)\right)-H\left(\phi^{\prime}\left(u_{+}(\tau(t))\right)\right)\right. \\
& \left.+\phi^{\prime}\left(u_{+}(\tau(t))\right)-\psi\left(\phi^{\prime}\left(u_{+}(\tau(t))\right)\right) H^{\prime}\left(\psi\left(\phi^{\prime}\left(u_{+}(\tau(t))\right)\right)\right)\right\} \\
& +\psi\left(\phi^{\prime}\left(u_{+}(\tau(t))\right)\right) H^{\prime}\left(\phi^{\prime}\left(\sigma_{-}(t)\right)-H\left(\psi\left(\phi^{\prime}\left(u_{+}(\tau(t))\right)\right)\right) .\right.
\end{aligned}
$$

By definition, we have

so that

$$
H^{\prime}\left(\psi\left(\phi^{\prime}\left(u_{+}(\tau(t))\right)\right)\right)=\frac{H\left(\phi^{\prime}\left(u_{+}(\tau(t))\right)\right)-H\left(\psi\left(\phi^{\prime}\left(u_{+}(\tau(t))\right)\right)\right)}{\phi^{\prime}\left(u_{+}(\tau(t))\right)-\psi\left(\phi^{\prime}\left(u_{+}(\tau(t))\right)\right)},
$$

$$
\tilde{y}^{\prime}(t, \sigma(t))=\psi\left(\phi^{\prime}\left(u_{+}(\tau(t))\right)\right) H^{\prime}\left(\phi^{\prime}(\sigma(t))\right)-H\left(\psi\left(\phi^{\prime}\left(u_{+}(\tau(t))\right)\right)\right) .
$$

Thus we have

$$
\begin{aligned}
\frac{d}{d t}\left(\tilde{y}(t, \sigma(t))-y_{-}(t, \sigma(t))\right)= & H^{\prime}\left(\phi^{\prime}\left(\sigma_{-}(t)\right)\right)-H\left(\psi\left(\phi^{\prime}\left(u_{+}(\tau(t))\right)\right)\right) \\
& -H^{\prime}\left(\phi^{\prime}(\sigma(t))\right)\left(\phi^{\prime}\left(\sigma_{-}(t)\right)-\psi\left(\phi^{\prime}\left(u_{+}(\tau(t))\right)\right)\right) .
\end{aligned}
$$

Since both $\phi^{\prime}\left(\sigma_{-}(t)\right)$ and $\psi\left(\phi^{\prime}\left(u_{+}(\tau(t))\right)\right)$ belong to the concave region of $H(p), \phi^{\prime}\left(\sigma_{-}(t)\right)$ $<\psi\left(\phi^{\prime}\left(u_{+}(\tau(t))\right)\right)$ for $t>t_{\alpha}$. Therefore

$$
\frac{H^{\prime}\left(\phi^{\prime}\left(\sigma_{-}(t)\right)\right)-H\left(\psi\left(\phi^{\prime}\left(u_{+}(\tau(t))\right)\right)\right.}{\phi^{\prime}\left(\sigma_{-}(t)\right)-\psi\left(\phi^{\prime}\left(u_{+}(\tau(t))\right)\right)}<H^{\prime}\left(\phi^{\prime}(\sigma(t))\right),
$$

hence we have $\frac{d}{d t}\left(\tilde{y}(t, \sigma(t))-y_{-}(t, \sigma(t))\right)>0$. Since $\tilde{y}\left(t_{\alpha}, \sigma\left(t_{\alpha}\right)\right)=y_{-}\left(t_{\alpha}, \sigma\left(t_{\alpha}\right)\right)$, the above inequality means that $\left.\tilde{y}(t, \sigma(t))>y_{-}(t, \sigma(t))\right)$ for $t>t_{\alpha}$. It follows that there exists a unique $\left(t, \chi_{r}(t)\right)$ with $x\left(t, u_{\alpha}\right)<\chi_{r}(t)<\sigma(t)$ such that $\tilde{y}\left(t, \chi_{r}(t)\right)=y_{-}\left(t, \chi_{r}(t)\right)$.
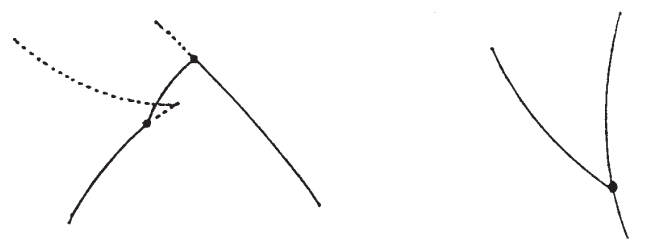

Fig. 10 
Then we can draw a picture of the graph of the viscosity solution for $t>t_{\alpha}$ and the bifurcation of the shock curves around $t_{\alpha}$ (cf. Figure 10).

For other cases, the detailed discussions will appear elsewhere.

\section{References}

[1] V. I. Arnol'd, S. M. Gusein-Zade and A. N. Varchenko, Singularities of Differentiable Maps, Birkhäuser, 1986.

[2] M. Bardi and L. C. Evans, On Hopf's formulas for solutions of Hamilton-Jacobi equations, Nonlinear Anal. 8 (1984), 1373-1389.

[3] P. Bernhard, Singular surfaces in differential games, an introduction, in: Differential Games and Applications, Lecture Notes in Control and Inform. Sci. 3, P. Hagedorn et al. (eds.), Springer, 1977, 1-33.

[4] A. Bogaevskiư, Modifications of singularities of minimum functions and bifurcations of shock waves at the Burgers equation with vanishing viscosity, Leningrad Math. J. 1 (1990), 807-823.

[5] M. G. Crandall, H. Ishii and P.-L. Lions, User's guide to viscosity solutions of second order partial differential equations, Bull. Amer. Math. Soc. 27 (1992), 1-67.

[6] M. G. Crandall and P.-L. Lions, Viscosity solutions of Hamilton-Jacobi equations, Trans. Amer. Math. Soc. 277 (1983), 1-42.

[7] M. G. Crandall, L. C. Evans and P.-L. Lions, Some properties of viscosity solutions of Hamilton-Jacobi equations, Trans. Amer. Math. Soc 282 (1984), 487-502.

[8] L. C. Evans and P. E. Souganides, Differential games and representation formulas for solutions of Hamilton-Jacobi-Isaacs equations, Indiana Univ. Math. J. 33 (1984), 773-797.

[9] W. H. Fleming and H. M. Soner, Controlled Markov Processes and Viscosity Solutions, Springer, 1993.

[10] V. A. Florin, Some simplest nonlinear problems of the consolidation of an aqueously saturated earthen medium, Izv. Akad. Nauk SSSR Otdel.Tekhn. Nauk 9 (1948), 13891397.

[11] E. Hopf, Generalized solution of non-linear equations of first order, J. Math. Mech. 14 (1965), 951-973.

[12] R. Isaacs, Differential Games, Wiley, New York, 1965.

[13] S. Izumiya, Geometric singularities for Hamilton-Jacobi equation, Adv. Stud. Pure Math. 22 (1993), 89-100.

[14] - The theory of Legendrian unfoldings and first order differential equations, Proc. Roy. Soc. Edinburgh 123A (1993), 517-532.

[15] S. Izumiya and G. T. Kossioris, Semi-local classification of geometric singularities for Hamilton-Jacobi equations, J. Differential Equations 118 (1995), 166-193.

[16] - - - Realization theorem of geometric singularities for Hamilton-Jacobi equations, preprint.

[17] G. T. Kossioris, Propagation of singularities for viscosity solutions of Hamilton-Jacobi equations in one space variable, Comm. Partial Differential Equations 18 (1993), 747770 .

[18] - Formation of singularities for viscosity solutions of Hamilton-Jacobi equations in higher dimensions, ibid., 1085-1108. 
[19] S. Nakane, Formation of shocks for a single conservation law, SIAM J. Math. Anal. 19 (1988), 1391-1408.

[20] - Formation of singularities for Hamilton-Jacobi equations in several space variables, J. Math. Soc. Japan 43 (1991), 89-100.

[21] H. Rund, The Hamilton-Jacobi Theory in the Calculus of Variations, D. Van Nostrand, London, 1966.

[22] M. Tsuji, Solution globale et propagation des singularités pour l'équation de HamiltonJacobi, C. R. Acad. Sci. Paris 289 (1979), 397-400.

[23] - Formation of singularities for Hamilton-Jacobi equation II, J. Math. Kyoto Univ. 26 (1986), 299-308.

[24] V. M. Zakalyukin, Reconstructions of fronts and caustics depending on a parameter and versality of mappings, Itogi Nauki, Contemporary Problems in Mathematics 22 (1983), 53-93. 